\title{
FRF Sensitivity-Based Damage Identification Using Linkage Modelling for Limited Sensor Arrays
}

\author{
VAN VU NGUYEN ${ }^{\dagger}$, JIANCHUN LI and EMRE ERKMEN \\ Centre for Built Infrastructure Research, University of Technology Sydney \\ 15 Broadway, Ultimo, NSW 2007, Australia \\ Van.V.Nguyen-1@student.uts.edu.au' \\ MEHRISADAT MAKKI ALAMDARI and ULRIKE DACKERMANN \\ School of Civil and Environmental Engineering, University of New South Wales \\ High Street, Kensington, NSW 2052, Australia
}

\begin{abstract}
This paper presents a novel method to localise and quantify damage in a jack arch structure by introducing a linkage modelling technique to overcome issues caused by having limited sensors. The main strategy in the proposed Frequency Response Function (FRF) based sensitivity model updating approach is to divide the specimen into partitions. The Young's modulus of each partition is then updated to detect stiffness reduction caused by damage. System Equivalent Reduction Expansion Process (SEREP) is used to reduce the full finite element (FE) model to a linkage model. The number of measured degrees of freedom (DOFs) is then expanded to the linkage model using the mass and stiffness matrices of the linkage model for the synthesis of interpolated FRFs. The FRF sensitivities are then formulated using the linkage model along with the interpolated FRFs to iteratively calculate the values of the updating parameters until convergence is achieved. The methodology and theory behind this procedure is discussed and verified using a numerical and experimental study. The successful implementation of this method has the potential to detect the location and severity of damage where sensor placement is limited.
\end{abstract}

\section{Introduction}

In the past couple of decades, Structural Health Monitoring (SHM) has received significant attention in the field of asset management, especially for ageing infrastructures. SHM systems generally aim to provide information on the health state of a structure, including detecting, localising and quantifying damage and deterioration. Numerous damage detection methods have been developed over the years $[1,2]$. Many of these methods involve the analysis of vibration data, including modal data, which is directly related to the physical properties of a structure. Hence, the characteristics of damage in a structure can be identified by analysing its vibration response.

Damage detection using model updating has received increasing attention in the past couple of decades. The model updating procedure reduces the discrepancy between the results obtained from a finite element (FE) model and the results measured from a physical structure by adjusting the modelling parameters until the analytical and the measured results are in agreement. This procedure is well suited to the aims of damage detection. Model updating utilising computational intelligence 
techniques have been used for damage detection by several researchers. Kang et al. [3] used an immunity enhanced particle swarm optimisation algorithm to detect damage in a simply supported beam. Hao and Xia [4] applied genetic algorithms to the model updating procedure to detect the stiffness reduction in elements of a cantilever beam and a portal frame structure. Perera and Torres [5] also used genetic algorithms to detect damage in a simply supported beam structure. While these methods excel in finding the global minimum of the discrepancies between the measured and analytical results, they generally require a large number of iterations, which can be problematic for larger models.

One of the most successful approaches to model updating is the sensitivity method, which requires less iterations to reach an updated model than computational intelligence methods. Mottershead et al. [6] provided a tutorial on how to update an FE model using the sensitivity method and demonstrated the procedure on a Lynx helicopter airframe. Sinha et al. [7] used eigenvalue sensitivities in their model updating procedure to estimate crack locations and sizes in a cantilever beam. Shi et al. [8] derived the sensitivity of modal strain energy with respect to damage and used this sensitivity to detect damage in a frame structure.

An important issue in SHM is the limited number of sensor arrays. In the ideal case, there would be one sensor corresponding to each degree of freedom (DOF) in the FE model. However, this is rarely possible in practical applications, due to the limited sensor availability and the inaccessibility of certain areas for sensor placement. The spatial incompleteness of measured data is commonly addressed by researchers by reducing the FE model to the number of measured sensors. Mousavi and Gandomi [9] addressed this issue by developing an iterative hybrid method that uses dynamic condensation to detect damage in structures with incomplete modal data. Hansen et al. [10] used Guyan reduction to condense their T-shaped FE model to ensure that the numerical DOFs corresponded with the measured DOFs. Sun and Büyüköztürk [11] used an iterated improved reduced system (IIRS) to reduce their FE model from 8 DOF to 4 DOF to match the measured data. Another added benefit of model reduction is the overall reduction in the computation time required for model updating. Weng et al. [12] derived eigenvalue and eigenvector partial derivatives with respect to a structural parameter based on a dynamic condensation technique to improve the efficiency of the model updating procedure.

The spatial incompleteness of measured data has also been address by researchers by expanding the measured eigenvectors to the FE model DOF. Au et al. [13] and Entezami et al. [14] used the System Equivalent Reduction Expansion Process (SEREP) by O'Callahan et al. [15] to expand their simulated measured data. Shi et al. [8] made adjustments to SEREP by incorporating a weighting coefficient that could be used to weight the accuracy of the analytical model and the measured data. The authors used this method to expand their measured mode shapes and localise damage in a frame structure.

The past investigations described in this paper used rod, beam and shell elements for their FE model updating procedure. However, in certain cases it is necessary to use solid elements to model a unique structure for damage detection, which can be computationally expensive. Hence, a linkage model that acts as an intermediary between a large FE model and a limited number of measured responses can be used for updating. In addition to expanding measured data, SEREP is also capable of reducing the full system matrices of a FE model with no loss of accuracy in the natural frequencies and mode shapes for the lower modes. Expansion can also be achieved by directly using the mass and stiffness matrices of a model which eliminates the need for eigenvalue analysis or mode tracking. 
Model updating can be achieved by reducing the discrepancies between the analytical and experimental FRFs directly. The FRFs contain information on the modal properties of a structure. This eliminates the need to pair the analytical to the experimental modes in each iteration of model updating. Alamdari et al. [16] used gradient-based design optimisation to reduce the discrepancies between analytical and measured FRFs by adjusting the non-linear properties of a joint. Araújo et al. [17] identified the damage in a laminated structure using the FRF sensitivity method. The authors also pointed out that the FRF sensitivity technique produced better results than the modal sensitivity method in their study due to the FRF sensitivity method having an overdetermined set of equations as opposed to the modal sensitivity method, which had an underdetermined set of equations.

This paper proposes the use of FE model updating using the FRF sensitivity method to localise and quantify damage in a replicated jack arch structure of the Sydney Harbour Bridge via a linkage model. Details of prior studies of the jack arch structure can be found in Mustapha et al. [18] and Nguyen et al. [19]. The parameters defined for the model updating procedure include the Young's modulus of 10 divided sections of the specimen and three additional parameters relating to the boundary conditions of the jack arch. The full FE model contains 5,706 DOFs and is reduced to a linkage model containing 60 DOFs using SEREP. The mode shapes determined experimentally using 11 accelerometers are then expanded to $60 \mathrm{DOFs}$ using the mass and stiffness matrices of the linkage model. The receptance FRFs are synthesised based on the expanded mode shapes and used to form the FRF sensitivity matrix and FRF residual vectors. The linear least squares method incorporating the trust region reflective algorithm is then used to update the parameters. This procedure is iteratively repeated until the convergence criterion is met. The damage location and severity is then determined based on the updated parameters. The procedure is verified numerically and experimentally.

The main innovation in the proposed method is that it uses the mass and stiffness matrices of a reduced FE model to expand the mode shapes obtained from the measured data. Past investigations that aimed to match the DOFs of the FE model to measured mode shapes have either reduced the FE model [9-12] or expanded the modal data [8, 13, 14]. If the DOFs of the FE model are reduced to the measured DOFs, then information of the mode shapes retained from the FE model can be insufficient. On the other hand, expanding the measured DOFs to the FE model can result in the model updating process being computationally expensive. This method aims to provide a balance between the information lost due to model reduction and the computational cost of expanding the measured DOF by introducing the linkage model.

\section{Addressing the Limited Sensor Issue Using a Linkage Model}

In real structures, it is impractical (if not impossible) to place one sensor at each location of all DOFs in the FE model. These restrictions are due to the limited number of available sensors for instrumentation and inaccessibility of certain areas of the structure. This study proposes the use of a linkage model that reduces the DOFs of a 3D FE model to a linkage model while expanding the measured DOFs to the same model. In this investigation, SEREP [15] is used for model reduction. The procedure requires partitioning of the mass $(\mathbf{M})$ and stiffness $(\mathbf{K})$ matrices according to the expression in Eq. (2.1).

$$
\mathbf{M}=\left[\begin{array}{cc}
\mathbf{M}_{\mathrm{mm}} & \mathbf{M}_{\mathrm{ms}} \\
\mathbf{M}_{\mathrm{sm}} & \mathbf{M}_{\mathrm{ss}}
\end{array}\right] \text { and } \mathbf{K}=\left[\begin{array}{cc}
\mathbf{K}_{\mathrm{mm}} & \mathbf{K}_{\mathrm{ms}} \\
\mathbf{K}_{\mathrm{sm}} & \mathbf{K}_{\mathrm{ss}}
\end{array}\right]
$$


where the subscript $m$ refers to the master DOF that will be retained and $\mathrm{s}$ is the slave DOF that will be removed after the reduction procedure. The transformation matrix, $\mathbf{T}$, used to reduce the FE model can be deduced using Eq. (2.2)

$$
\mathbf{T}=\left[\begin{array}{c}
\boldsymbol{\Phi}_{\mathrm{m}} \\
\boldsymbol{\Phi}_{\mathrm{s}}
\end{array}\right] \boldsymbol{\Phi}_{\mathrm{m}}^{+}
$$

where $\boldsymbol{\Phi}$ is the matrix containing the mass normalised eigenvectors of the FE model. The superscript + refers to the pseudo inverse of a matrix. The transformation matrix is then used to reduce the full system matrices to the linkage model system matrices using Eq. (2.3)

$$
\mathbf{M}_{\mathbf{l}}=\mathbf{T}^{\mathrm{T}} \mathbf{M} \mathbf{T} \text { and } \mathbf{K}_{\mathbf{l}}=\mathbf{T}^{\mathrm{T}} \mathbf{K} \mathbf{T}
$$

where $\mathbf{M}_{\mathbf{l}}$ and $\mathbf{K}_{\mathbf{l}}$ are the mass and stiffness matrices of the linkage model. The system matrices of the linkage model are then used to expand the measured DOFs to the linkage model DOFs. Friswell et al. [20] provide the procedure for this using Eq. (2.4)

$$
\left(-\omega_{\mathrm{j}}^{2}\left[\begin{array}{cc}
\mathbf{M}_{\mathbf{l}_{\mathrm{mm}}} & \mathbf{M}_{\mathbf{l}_{\mathrm{ms}}} \\
\mathbf{M}_{\mathbf{l}_{\mathrm{sm}}} & \mathbf{M}_{\mathbf{l}_{\mathrm{ss}}}
\end{array}\right]+\left[\begin{array}{cc}
\mathbf{K}_{\mathbf{l}_{\mathrm{mm}}} & \mathbf{K}_{\mathbf{l}_{\mathrm{ms}}} \\
\mathbf{K}_{\mathbf{l}_{\mathrm{sm}}} & \mathbf{K}_{\mathbf{l}_{\mathrm{ss}}}
\end{array}\right]\right)\left\{\begin{array}{l}
\boldsymbol{\phi}_{\mathrm{mj}} \\
\boldsymbol{\phi}_{\mathrm{sj}}
\end{array}\right\}=\left\{\begin{array}{l}
\mathbf{0} \\
\mathbf{0}
\end{array}\right\}
$$

where $\boldsymbol{\phi}_{\mathrm{mj}}$ and $\boldsymbol{\phi}_{\mathrm{sj}}$, respectively, represent the mode shape at the measured and unmeasured DOFs of the $\mathrm{j}^{\text {th }}$ mode and $\boldsymbol{\omega}_{\mathbf{j}}^{2}$ is the corresponding eigenvalue. The bottom half of this equation can be rearranged to Eq. (2.5)

$$
\boldsymbol{\phi}_{\mathrm{sj}}=-\left(-\omega_{\mathrm{j}}^{2} \mathbf{M}_{\mathbf{l}_{\mathrm{ss}}}+\mathbf{K}_{\mathbf{l}_{\mathrm{ss}}}\right)^{-1}\left(-\omega_{\mathrm{j}}^{2} \mathbf{M}_{\mathbf{l}_{\mathrm{sm}}}+\mathbf{K}_{\mathbf{l}_{\mathrm{sm}}}\right) \boldsymbol{\phi}_{\mathrm{mj}}
$$

which can be used to calculate the interpolated mode shapes for the unmeasured DOFs using the mass and stiffness matrices of the linkage model and the measured eigenvectors. The interpolated mode shapes can be used to synthesise the receptance FRFs at the unmeasured DOFs using a formulation provided by Maia and Silva [21] expressed in Eq. (2.6)

$$
\alpha_{\mathrm{jk}}(\omega)=\sum_{\mathrm{r}=1}^{\mathrm{N}}\left(\frac{\phi_{\mathrm{jr}} \phi_{\mathrm{kr}}}{\mathrm{i} \omega-\mathrm{s}_{\mathrm{r}}}+\frac{\phi_{\mathrm{jr}}^{*} \phi_{\mathrm{kr}}^{*}}{\mathrm{i} \omega-\mathrm{s}_{\mathrm{r}}^{*}}\right)
$$

where there is $\mathrm{N}$ number of modes used in the FRF synthesis, $\boldsymbol{\phi}_{\mathbf{j r}}$ represents the modal displacement at response $\mathrm{j}$ for mode $\mathrm{r}, \boldsymbol{\phi}_{\mathrm{kr}}$ represents the modal displacement at the force input $\mathrm{k}$ for mode $\mathrm{r}$. The eigenvalues $\mathbf{S}_{\mathbf{r}}$ are the poles for mode $\mathbf{r}$. The residuals in Eq. (2.6) are normalised by multiplying the numerators with Eq. (2.7)

$$
\mathrm{a}_{\mathrm{r}}=\frac{1}{2 \mathrm{im}_{\mathrm{r}} \omega_{\mathrm{dr}}}
$$

where $i=\sqrt{-1}, \mathbf{m}_{\mathbf{r}}$ is the modal mass and $\boldsymbol{\omega}_{\mathbf{d r}}$ is the damped natural frequency of mode $\mathrm{r}$. The synthesised FRFs can be used in the model updating procedure covered in the next section of this paper. 


\section{Damage Identification Using FRF Sensitivity Model Updating}

The location and severity of damage is identified in this study by updating FE model using FRF sensitivities. The method is based on the iterative formulation of the FRF sensitivities derived by Lin and Ewins [22]. In each iteration, this method uses the Linear Least Squares Solver "Isqlin" in MATLAB to minimise the squared 2-norm of the residual expressed in Eq. (2.8)

$$
\min _{\Delta \mathbf{p}} \frac{1}{2}\|\mathbf{S} \Delta \mathbf{p}-\Delta \boldsymbol{\alpha}\|_{2}^{2}
$$

where $\Delta \mathbf{p}$ is the vector of updating parameters and $\Delta \boldsymbol{\alpha}$ is the vector of FRF residuals calculated using Eq. (2.9)

$$
\Delta \boldsymbol{\alpha}=\left\{\begin{array}{c}
\left\{\boldsymbol{\alpha}_{X}\left(\omega_{1}\right)\right\}_{j}-\left\{\boldsymbol{\alpha}_{A}\left(\omega_{1}\right)\right\}_{j} \\
\vdots \\
\left\{\boldsymbol{\alpha}_{X}\left(\omega_{n}\right)\right\}_{j}-\left\{\boldsymbol{\alpha}_{A}\left(\omega_{n}\right)\right\}_{j}
\end{array}\right\}
$$

where $\left\{\boldsymbol{\alpha}_{X}\left(\omega_{i}\right)\right\}_{j}$ refers to the vector of the experimentally obtained receptance FRFs at the $\mathrm{i}^{\text {th }}$ measured frequency with each row of the vector corresponding to the DOF of the measured response with the input applied at coordinate $\mathbf{j}$ and $\left\{\boldsymbol{\alpha}_{A}\left(\omega_{i}\right)\right\}_{j}$ being the analytical counterpart. $\mathbf{S}$ is the FRF sensitivity matrix and can be calculated using Eq. (2.10)

$$
\mathbf{S}=\left[\begin{array}{ccc}
-\left[\boldsymbol{\alpha}_{\mathrm{A}}\left(\omega_{1}\right)\right]\left[\frac{\partial \mathbf{Z}_{\mathrm{A}}\left(\omega_{1}\right)}{\partial \mathrm{p}_{1}}\right]\left\{\boldsymbol{\alpha}_{\mathrm{X}}\left(\omega_{1}\right)\right\}_{\mathrm{j}} & \cdots & -\left[\boldsymbol{\alpha}_{\mathrm{A}}\left(\omega_{1}\right)\right]\left[\frac{\partial \mathbf{Z}_{\mathrm{A}}\left(\omega_{1}\right)}{\partial \mathrm{p}_{\mathrm{Np}}}\right]\left\{\boldsymbol{\alpha}_{\mathrm{X}}\left(\omega_{1}\right)\right\}_{\mathrm{j}} \\
\vdots & \ddots & \\
-\left[\boldsymbol{\alpha}_{\mathrm{A}}\left(\omega_{\mathrm{n}}\right)\right]\left[\frac{\partial \mathbf{Z}_{\mathrm{A}}\left(\omega_{\mathrm{n}}\right)}{\partial \mathrm{p}_{1}}\right]\left\{\boldsymbol{\alpha}_{\mathrm{X}}\left(\omega_{\mathrm{n}}\right)\right\}_{\mathrm{j}} & \cdots & -\left[\boldsymbol{\alpha}_{\mathrm{A}}\left(\omega_{\mathrm{n}}\right)\right]\left[\frac{\partial \mathbf{Z}_{\mathrm{A}}\left(\omega_{\mathrm{n}}\right)}{\partial \mathbf{p}_{\mathrm{Np}}}\right]\left\{\boldsymbol{\alpha}_{\mathrm{X}}\left(\omega_{\mathrm{n}}\right)\right\}_{\mathrm{j}}
\end{array}\right]
$$

where $\mathbf{Z}_{\mathrm{A}}(\omega)$ is the dynamic stiffness matrix expressed in the frequency domain in Eq. (2.11)

$$
\mathbf{Z}_{\mathbf{A}}(\omega)=\mathbf{K}+\mathrm{i} \omega \mathbf{C}-\omega^{2} \mathbf{M}
$$

where $\mathbf{K}$ is the stiffness matrix, $\mathbf{C}$ is the damping matrix and $\mathbf{M}$ is the mass matrix of the FE model. The formulation of the sensitivity matrix $\mathbf{S}$ requires the partial derivatives of the dynamic stiffness matrix with respect to the updating parameters to be calculated. The dynamic stiffness matrix derivatives can be reduced to the linkage model using the transformation matrix calculated in Eq. (2.2). Also, the numerical conditioning of Eq. (2.8) can be improved by normalising the updating parameters so that the current parameter estimate is unity. The choice of updating parameters depends on the purpose of the model updating procedure. For damage detection purposes, this is typically the Young's modulus of an element or a partition of the model. A decrease in the stiffness of the material can indicate damage in a structure. Dividing the FE model into partitions and using the Young's modulus of each partition as an updating parameter can be used as an approach for damage detection via model updating. The decision of DOFs to retain after model reduction can be tailored around the updating parameters chosen. An example of this is provided in the next section of this paper. The updated parameters can be used to form a damage index (DI) for each partition of the structure based on the overall stiffness reduction of each partition as expressed in Eq. (2.12)

$$
D I=\frac{\left|E_{h}-E_{d}\right|}{E_{h}}
$$


where $E_{h}$ and $E_{d}$ refer to the Young's modulus of a partition of the FE model in its healthy state and damaged state respectively. The model updating procedure is illustrated in Figure 1. In this investigation, this procedure is first applied to the specimen in its healthy state to capture the overall Young's modulus, $\mathrm{E}_{\mathrm{h}}$, and the stiffness of its boundary conditions before any damage occurs. Once the structure is damaged, it is partitioned so that the Young's Modulus, $\mathrm{E}_{\mathrm{d}}$, can be identified for each partition. The procedure in Figure 1 is repeated for each damage case. As indicated in the flow chart, the damage index is only calculated when the procedure is applied to the structure in its damaged state. For all model updating procedures applied in this investigation, the convergence criterion is set such that convergence is achieved when either the changes in all parameters is less than $0.1 \%$ or a maximum of 20 iterations is reached.

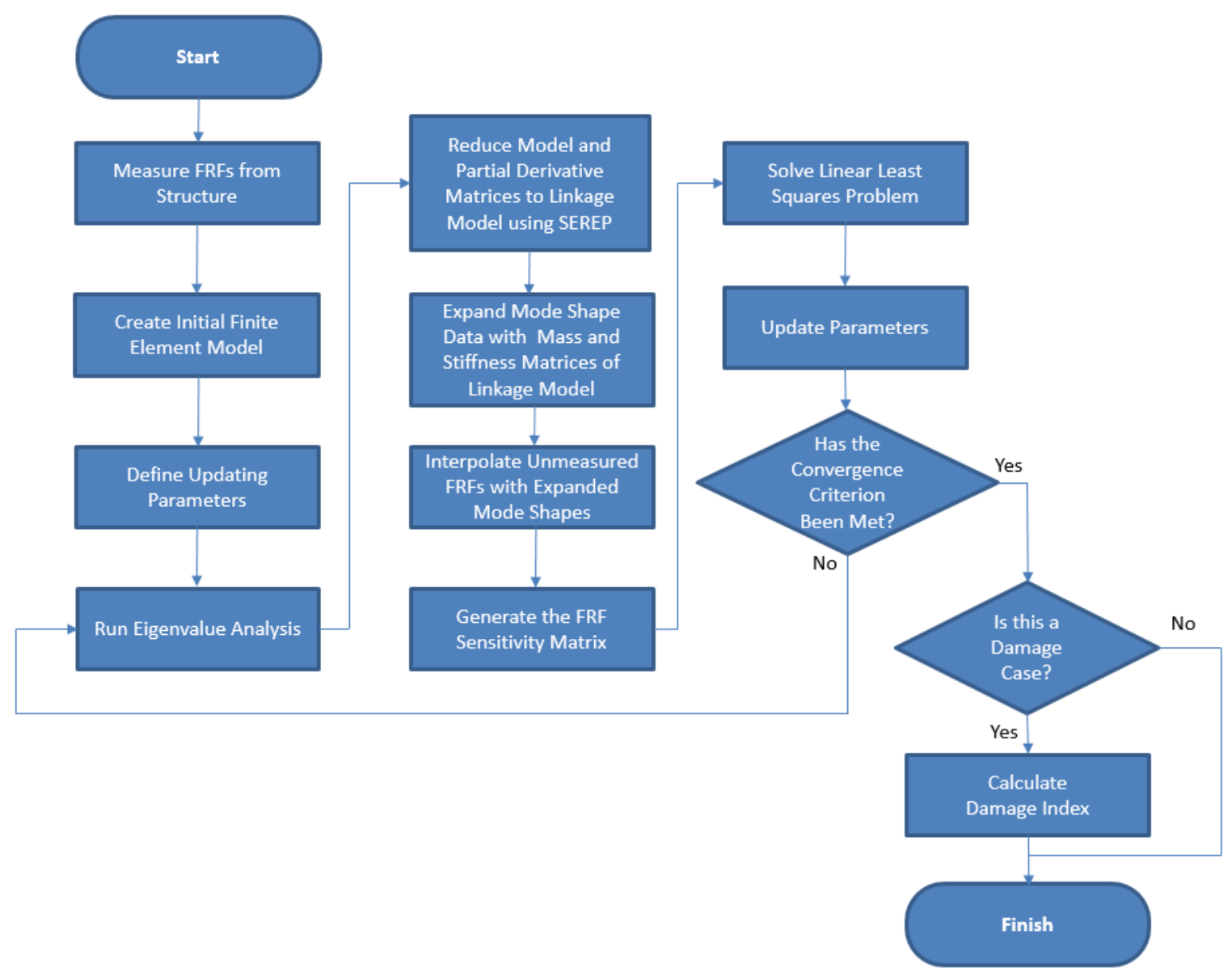

Fig. 1. Flow chart summarising the model updating procedure. 


\section{Case Study - Experimental Testing}

An experimental specimen replicating a jack arch component from the bus lane of the Sydney Harbour Bridge, shown in Figure 2, was used as the case study for this investigation. The jack arch component was $375 \mathrm{~mm}$ in height, $1,000 \mathrm{~mm}$ in width and 2,000 $\mathrm{mm}$ in length and had an internal steel I-Beam embedded along it. The cantilever structure was adhered to a clamp at the fixed end using plaster and was additionally supported at $1,000 \mathrm{~mm}$ from the front of the specimen.

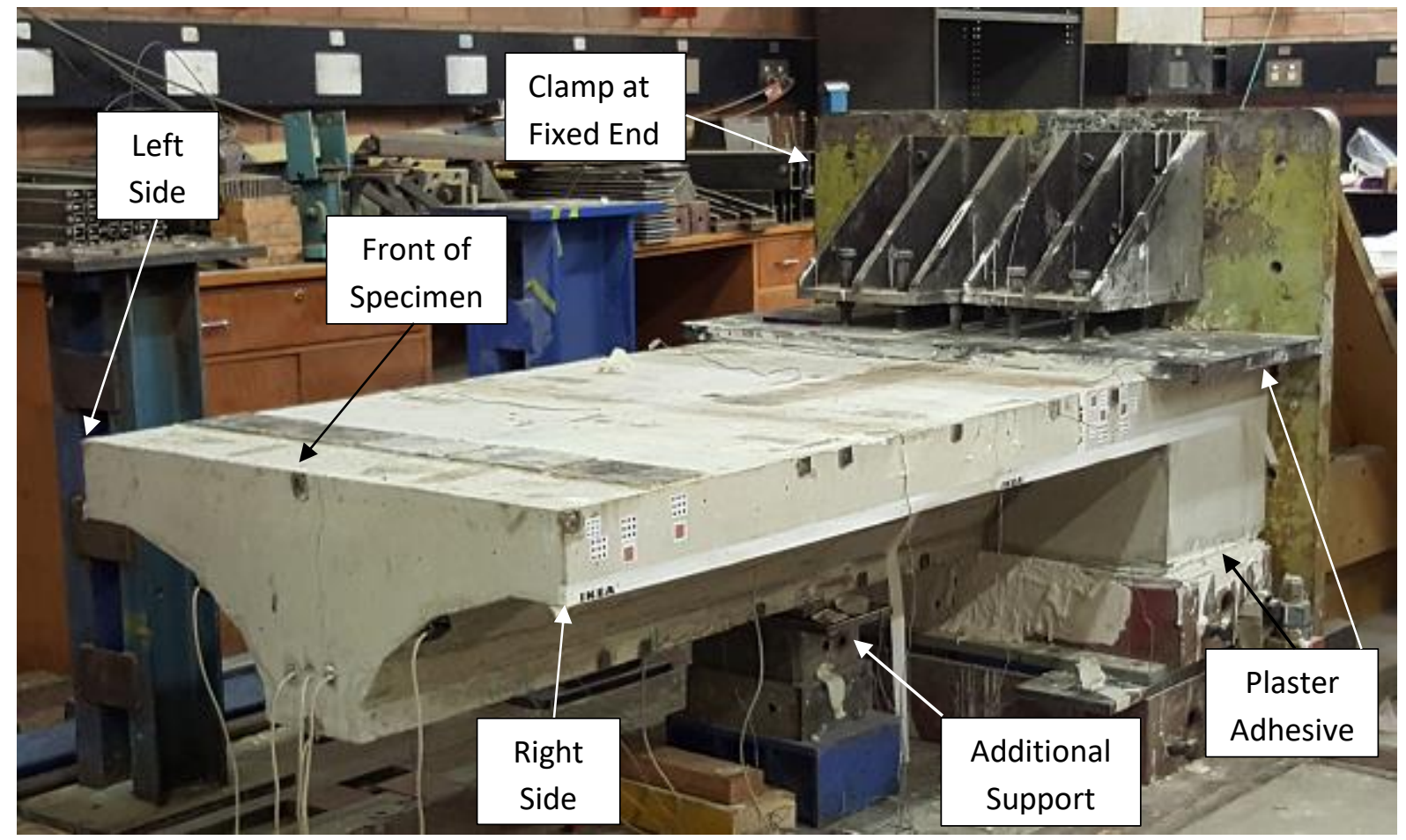

Fig. 2. Experimental jack arch specimen and its boundary condition setup.

Experimental modal analysis (EMA) was conducted on the specimen to extract its dynamic characteristics. An impact hammer was used to excite the structure 25 times to generate multiple data samples and eleven accelerometers (model PCB 352C34), A1 to A11, were used to measure the acceleration response of the structure in the vertical direction. Figure 3 shows the EMA testing set up. The data was collected with a sampling rate of $20 \mathrm{kHz}$ captured over 2 seconds for each impact. The time history and the auto-spectrum measured from an impact sample are illustrated in Figure 4. In general, the force level is relatively constant until a cut-off frequency is met, which is usually taken to be $10 \mathrm{~dB}$ below the maximum power [20]. In this example, the cut off frequency is taken to be $1,250 \mathrm{~Hz}$. A1 was chosen as the driving point FRF, since impacting the specimen near A1 was found to excite identifiable vertical bending, in-plane bending and torsional modes. The inertance FRFs for each impact sample were calculated and the outliers were removed. The remaining samples were averaged to emphasise the repeatable features of the FRFs while softening the non-repeatable features. The receptance FRFs were then calculated by dividing the inertance FRFs by $-\omega^{2}$ at each measured frequency.

The next step of this investigation was to introduce damage to the specimen. Therefore, a static load was applied at the front of the specimen using a hydraulic jack as shown in Figure 5. The specimen was treated as an overhanging cantilever beam in this configuration. The load was applied until an immediate reduction in the load carrying capacity of the structure was observed. After the loading was 
stopped, the hydraulic jack was removed and the dynamic test was repeated. A crack was observed at $1,300 \mathrm{~mm}$ measured on the left and 1,400 $\mathrm{mm}$ measured on the right of the specimen from its front with a depth of $275 \mathrm{~mm}$ as shown in Figure 6. The specimen was loaded a second time and the depth of the crack was increased to $300 \mathrm{~mm}$. Modal testing was conducted for the two damage cases.

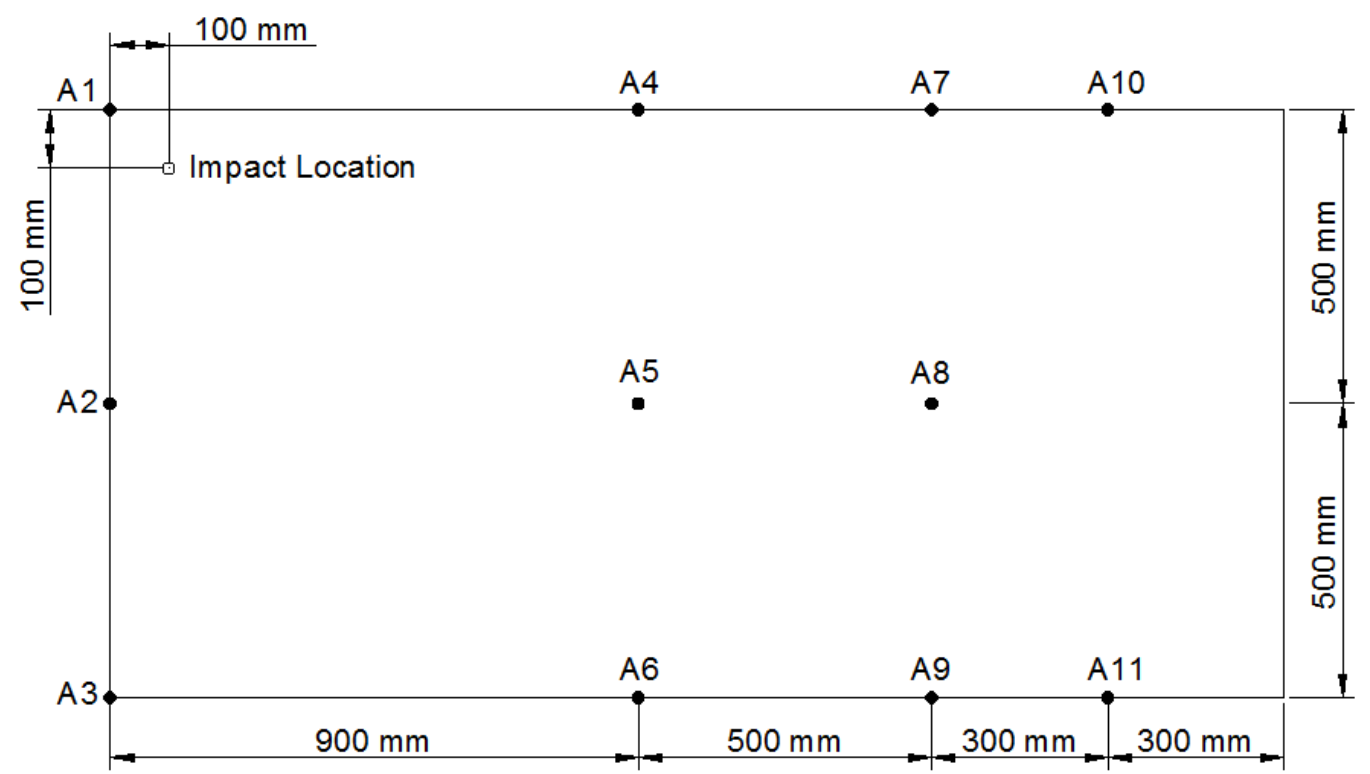

Fig. 3. Schematic for dynamic testing showing the locations of the hammer impact and the accelerometers A1 to A11 measuring the vertical acceleration.

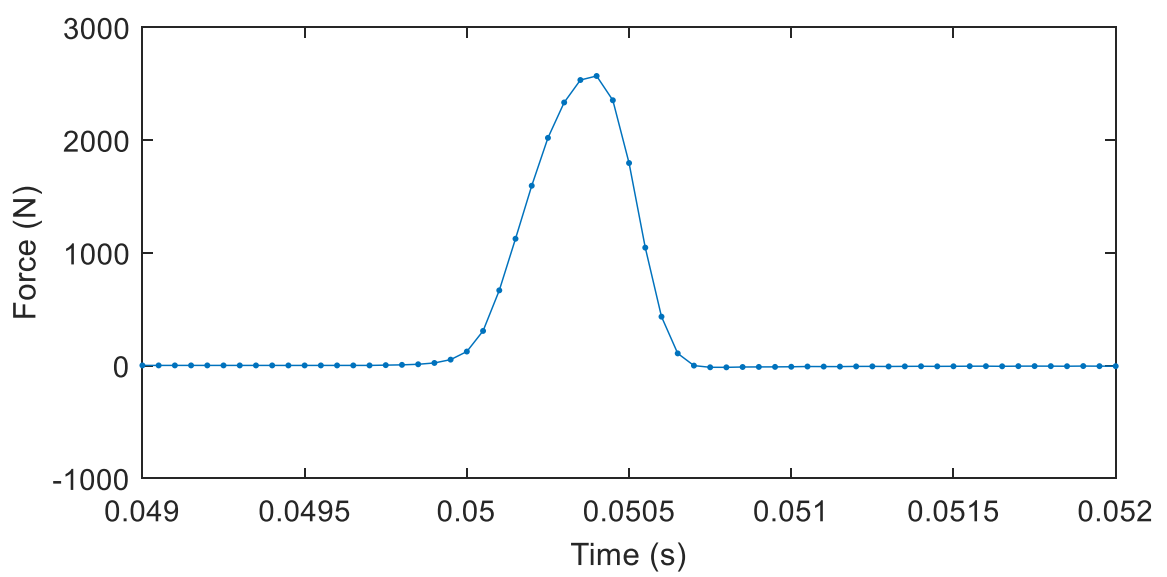

(a)

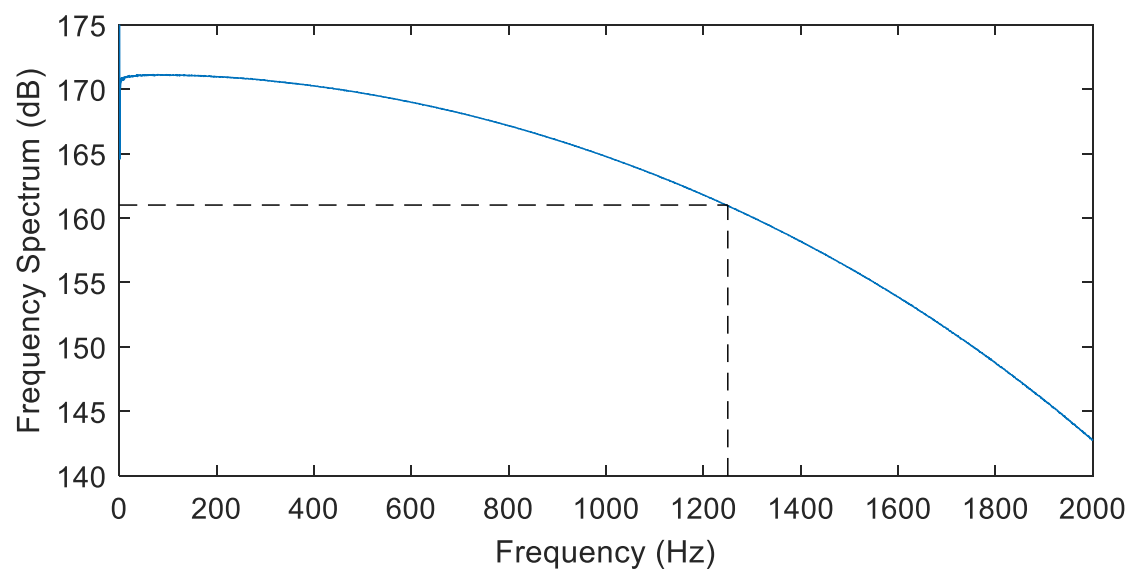

(b)

Fig. 4: The (a) time history and (b) auto-spectrum measured from the force of an impact sample. 


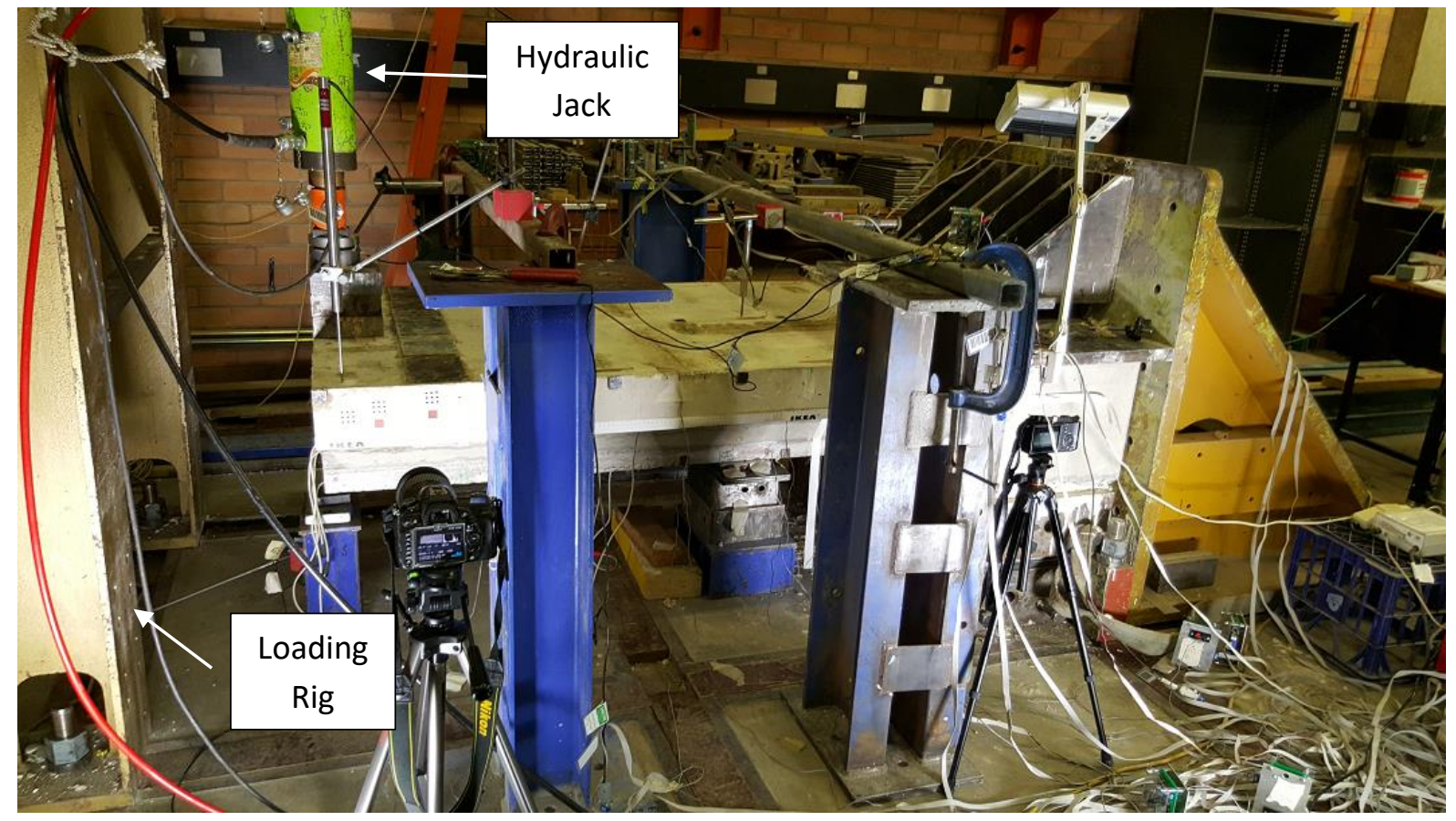

Fig. 5. Setup for static load testing.
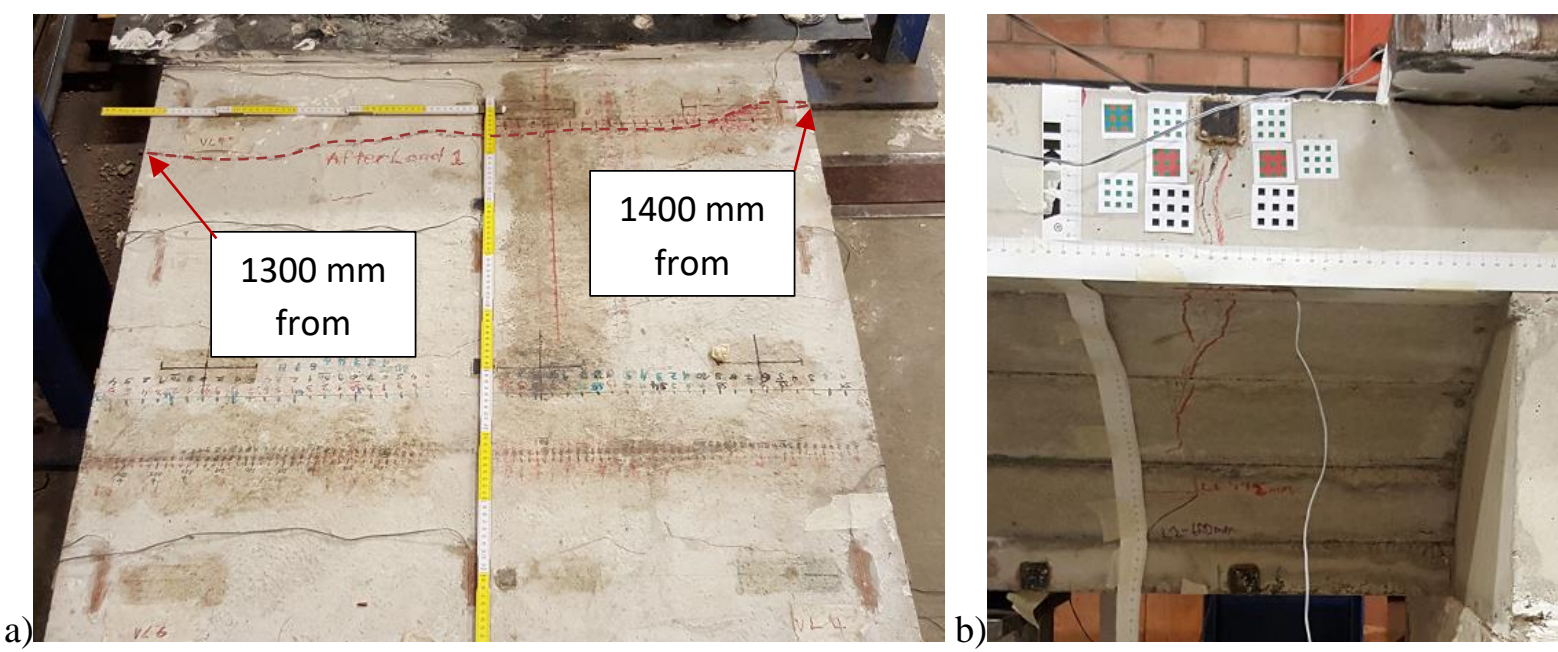

Fig. 6. A single crack observed from (a) top view and (b) right side view.

\section{Numerical Validation}

An FE model was constructed in ANSYS APDL using 20 noded SOLID 186 elements to exhibit quadratic displacement behaviour in the model. This model is illustrated in Figure 7. The updating parameters in the FE model were defined by dividing the model into ten partitions along the structure with each partition being $200 \mathrm{~mm}$ long. This was done to detect the location and severity of the transverse crack that was expected to occur as a result of loading the specimen at its tip in the overhanging cantilever beam configuration shown in Figure 5. A Young's modulus value was defined for each of these partitions. These parameters are denoted as P1 to P10 in Figure 7. The initial Young's modulus for the concrete specimen was set to $30 \mathrm{GPa}$. The Young's modulus of the adhesives connecting the specimen to the supports were also considered as updating parameters, as they had a significant influence on the dynamic properties of the structure. They are denoted as BC1 
to $\mathrm{BC} 3$ in Figure 7. The partial derivatives of the mass and stiffness matrices with respect to the updating parameters were numerically determined by perturbing the updating parameters by a value of $1 \mathrm{MPa}$ and calculating the differences in the perturbed and unperturbed system matrices.

Memory constraints were a major issue in the numerical modelling of the specimen. The mass and stiffness matrices of the FE model were created using ANSYS APDL. However, the matrix operations described in the previous sections of this paper were conducted in MATLAB. For the proposed procedure, it was necessary to store multiple system matrices including the mass and stiffness matrices of the FE model with its current parameter estimates and the partial derivatives of the system matrices with respect to the updating parameters. The full FE model contained 5,706 DOF. It was observed that a maximum of 5.2 GB of random-access memory (RAM) was used during the updating procedure. If the mesh density was to double in any dimension, then the computation would require four times the memory. This was problematic, as the system used to conduct this analysis contained $16 \mathrm{~GB}$ of RAM. Hence the quality of the model needed to be compromised to demonstrate the proposed method. For example, the plaster used to adhere the specimen to the supports was modelled with elements that had a poor aspect ratio and the steel I-beam was excluded from the FE model. In future works, strategies to improve memory management will be employed so that higher quality models can be used.

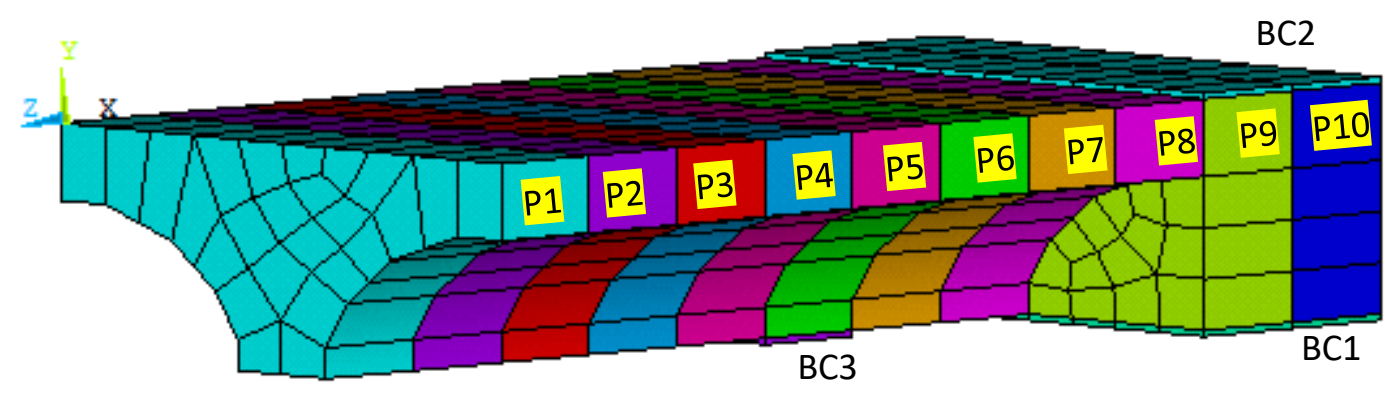

Fig. 7. Finite element model of jack arch specimen.

The numerical validation demonstrates the model updating procedure being applied to the structure in its damaged state. Smeared cracking was used to simulate damage by reducing the Young's modulus of P7, as the crack identified on the specimen was located within the region covered by this parameter. The Young's modulus of P7 was arbitrarily reduced to $20 \mathrm{GPa}$ to test whether the damage identification method could detect this stiffness reduction. Eleven receptance FRFs were synthesised based on the experimental setup of the modal test shown in Figure 3. Rayleigh damping was assumed in the FRF synthesis with the values of $\alpha=5.95$ and $\beta=1.1 \times 10^{-6}$, which were based on the first and last modes of interest of the specimen in its healthy state. The eleven receptance FRFs were then used as the measured data in the numerical study. In the initial iteration of the model updating procedure, the mass and stiffness matrices of the FE model were reduced from the 5,706 DOF shown in Figure 7 to the 60 DOF linkage model shown in Figure 8 using SEREP. The DOFs of the linkage model were chosen based on the updating parameters of the FE model. The partitions of the FE model covered a length of $200 \mathrm{~mm}$ along the specimen. Hence, $200 \mathrm{~mm}$ was chosen as the distance between the DOFs along the linkage model. The spacing of $250 \mathrm{~mm}$ across the specimen was chosen to retain a good aspect ratio for the grid and to ensure that there was a node in the centreline of the structure. This resulted in 55 DOFs for the linkage model. An additional 5 DOFs were added to include measured DOFs, resulting in a total of 60 DOFs for the linkage model. Only the vertical DOFs were considered for the linkage model since vibration was only measured in the vertical direction on the specimen. 


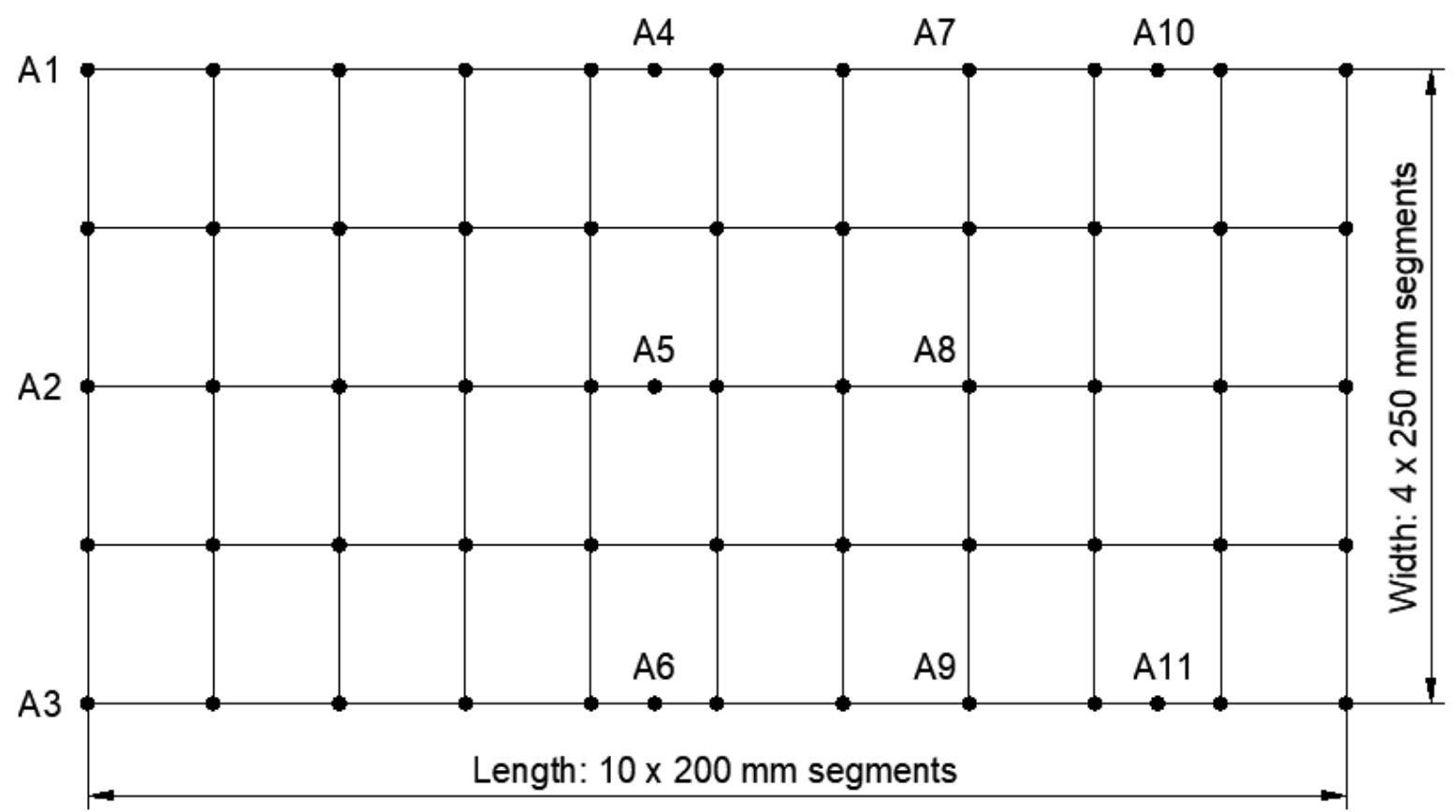

Fig. 8. Linkage FE model.

The mass and stiffness matrices of the linkage model were then used to expand the measured mode shapes to match the DOFs of the linkage model using Eq. (2.5). Examples of expanded mode shapes are shown in Figure 9. The interpolated receptance FRFs used in the updating algorithm were then synthesised using the expanded mode shapes with the expression in Eq. (2.6). The choice of measured frequencies was considered in the updating process. Sufficient measured frequencies were needed to ensure that there was adequate information on the dynamic properties of the structure. However, it was found that using too many measured frequencies resulted in the updating parameters being adjusted minimally with each iteration. According to Imregun et al. [23], there is a cut-off in the number of measured frequencies used where using more measured frequencies is no longer beneficial as the number of iterations needed to reach convergence is significantly increased.

The frequency range chosen for the updating procedure was $0 \mathrm{~Hz}$ to $1000 \mathrm{~Hz}$ with an increment of $2 \mathrm{~Hz}$. The next step of the damage detection method was to produce the FRF sensitivity matrix using Eq. (2.10). The sensitivity matrix consisted of 13 columns corresponding to the updating parameters and 60,120 rows to accommodate the 501 measured frequency points, 60 measurement locations including the measured and interpolated DOFs and the splitting of the real and imaginary components of the FRF. The linear least-squares problem in Eq. (2.8) was then solved using the linear leastsquares solver function in MATLAB with the trust region reflective algorithm. The lower bounds and upper bounds for P1 to P10 were set to $1 \mathrm{GPa}$ and $30 \mathrm{GPa}$, respectively. A value of $1 \mathrm{GPa}$ was used as a lower limit to allow the Young's Modulus of each partition to drop significantly to indicate its damage severity. $30 \mathrm{GPa}$ was used as the upper limit, as it was understood that the Young's modulus of each partition would not rise above its healthy value. This procedure was repeated iteratively with the linkage model system matrices and interpolated FRFs changing with each iteration according to the adjustments in the updating parameters. The convergence criteria to set to stop the iterative procedure, once either, all values had a change of less than $0.1 \%$, or a maximum of 20 iterations was reached. This criterion was met after 17 iterations. The comparison of the FRFs before and after 
updating is shown in Figure 10 and the parameter convergence is shown in Figure 11. The finalised updating parameters were used to calculate the damage index using Eq. (2.12). The results are summarised in Figure 12.
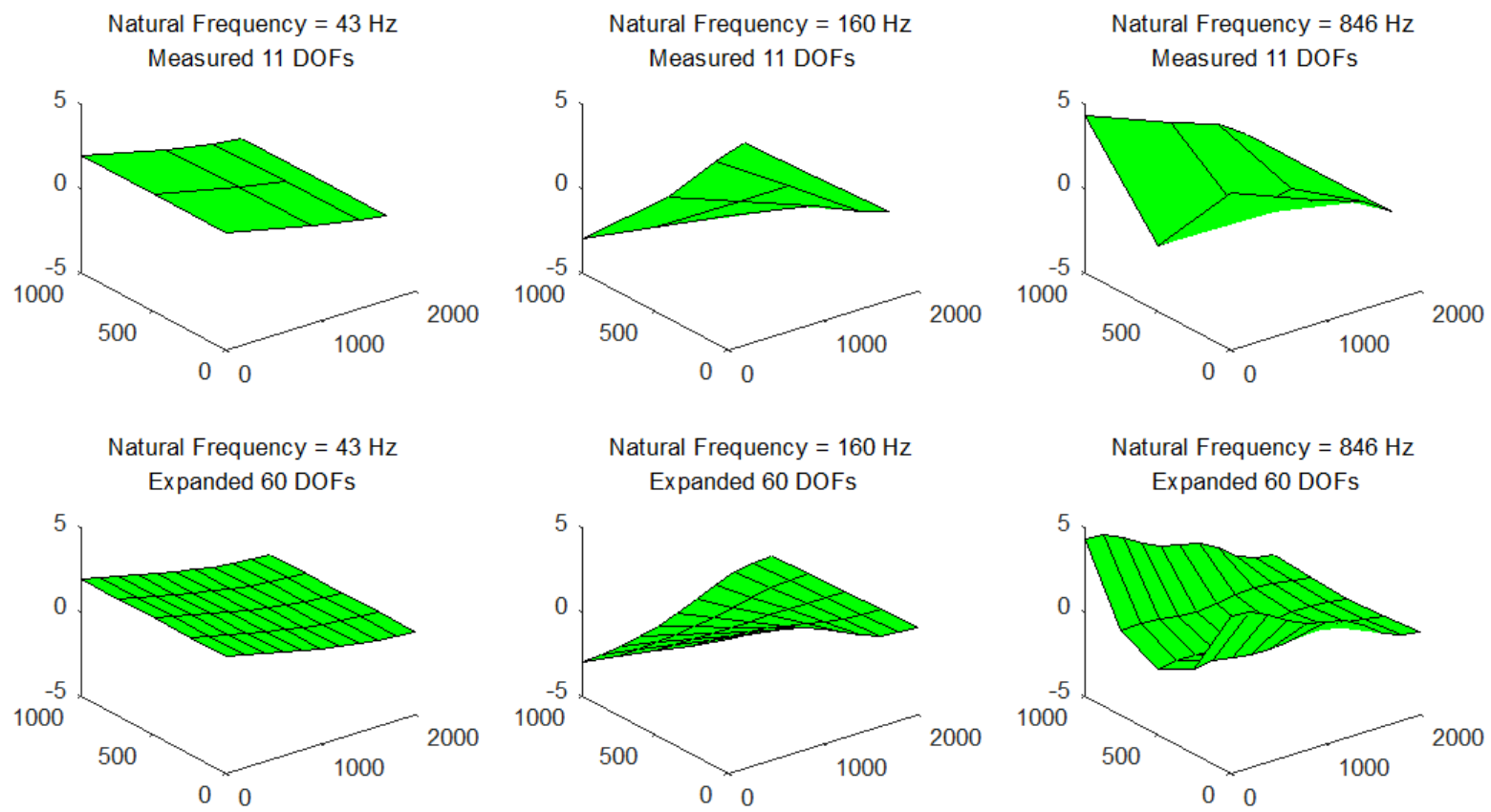

Fig. 9. Examples of mode shapes expanded from 11 DOFs to 60 DOFs for the numerical study.

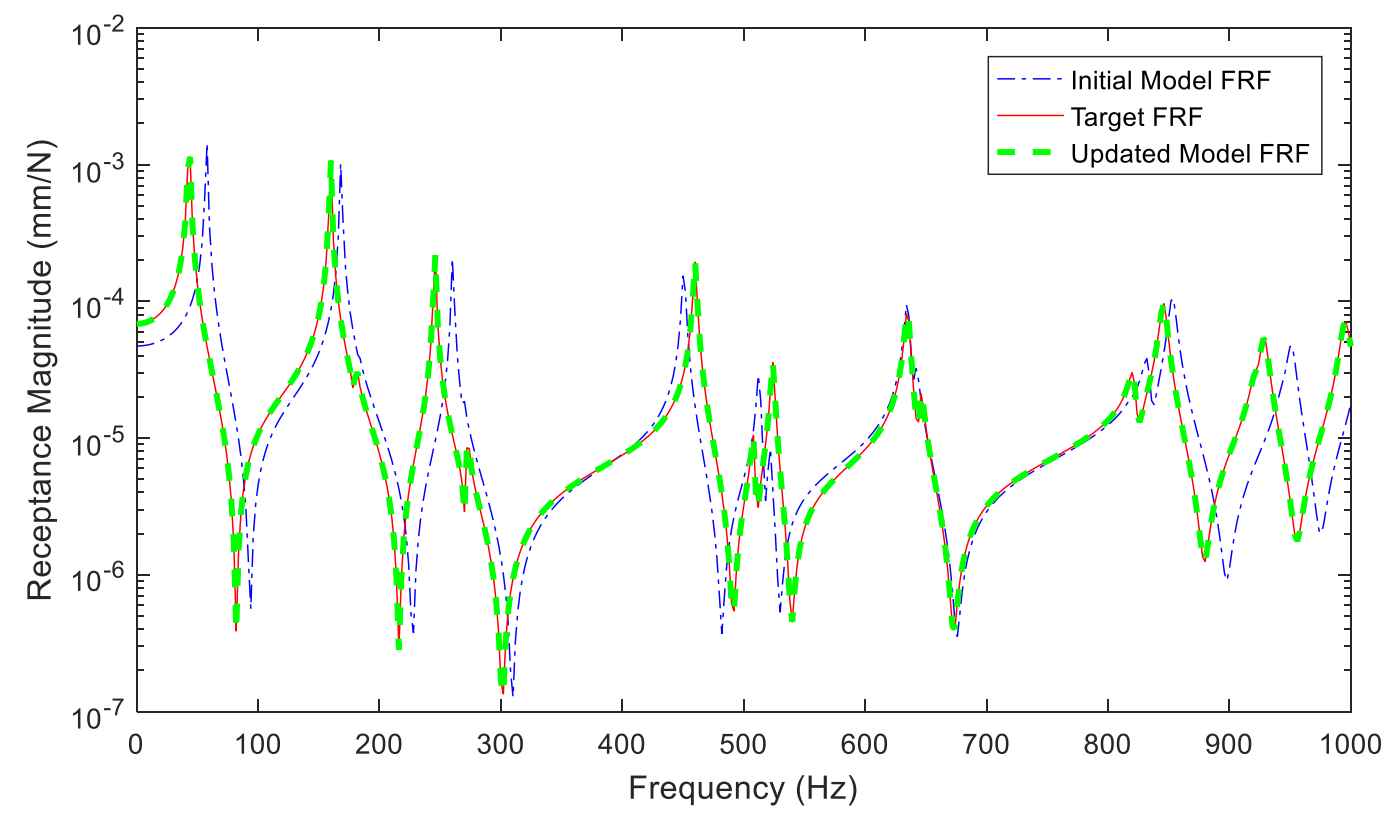

Fig. 10. Driving point FRF before and after updating in numerical investigation. 


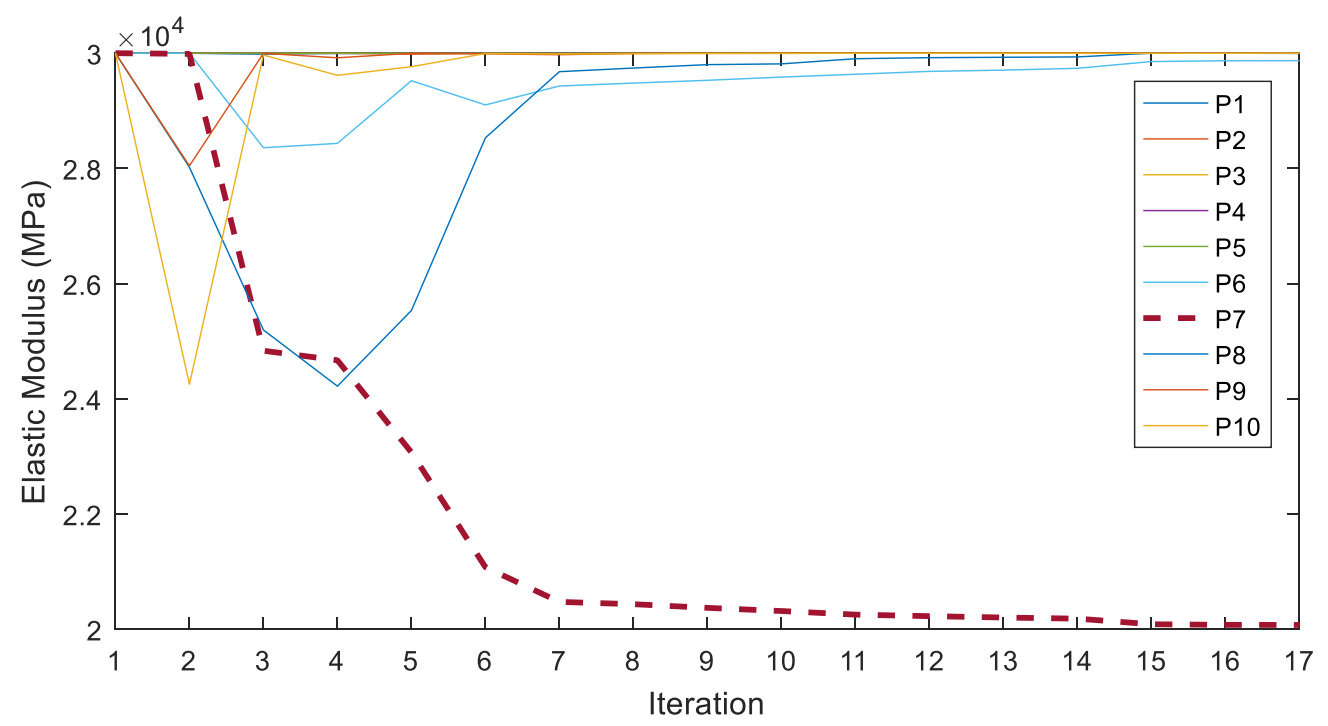

a)

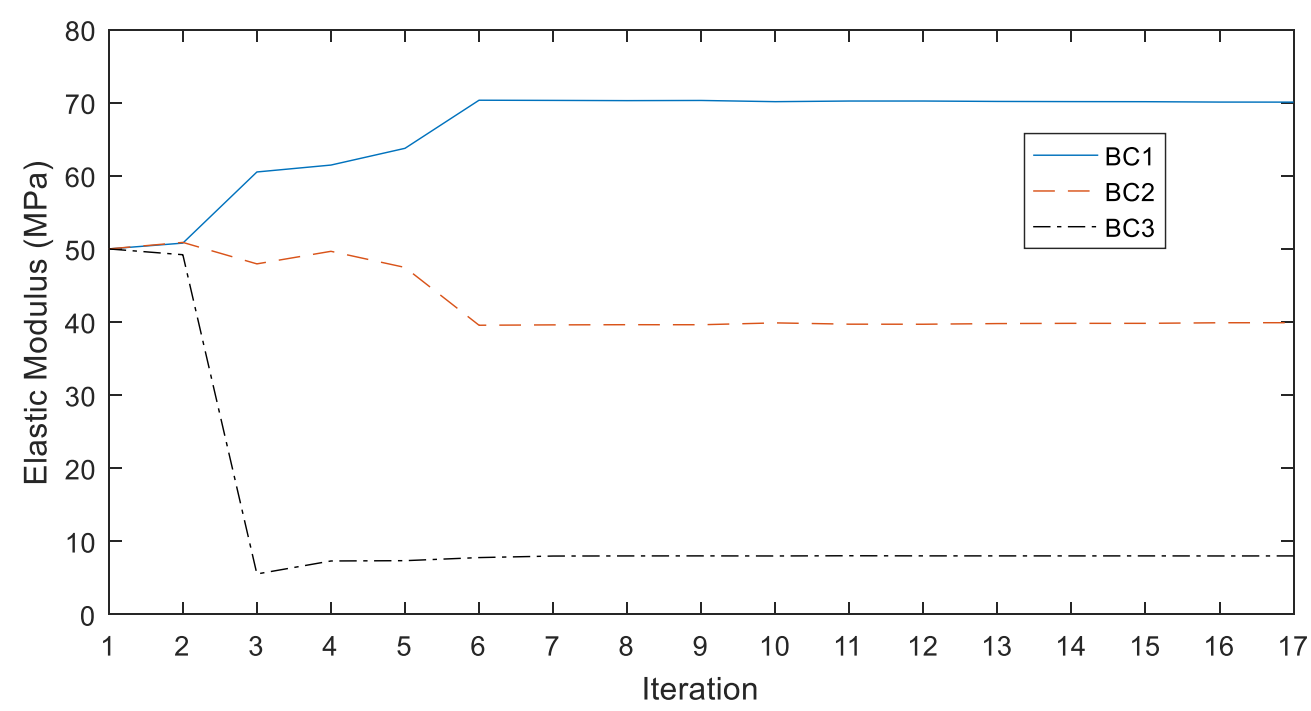

b)

Fig. 11. Convergence of updating parameters of numerical investigation for the elastic modulus of (a) specimen partitions and (b) boundary condition adhesives.

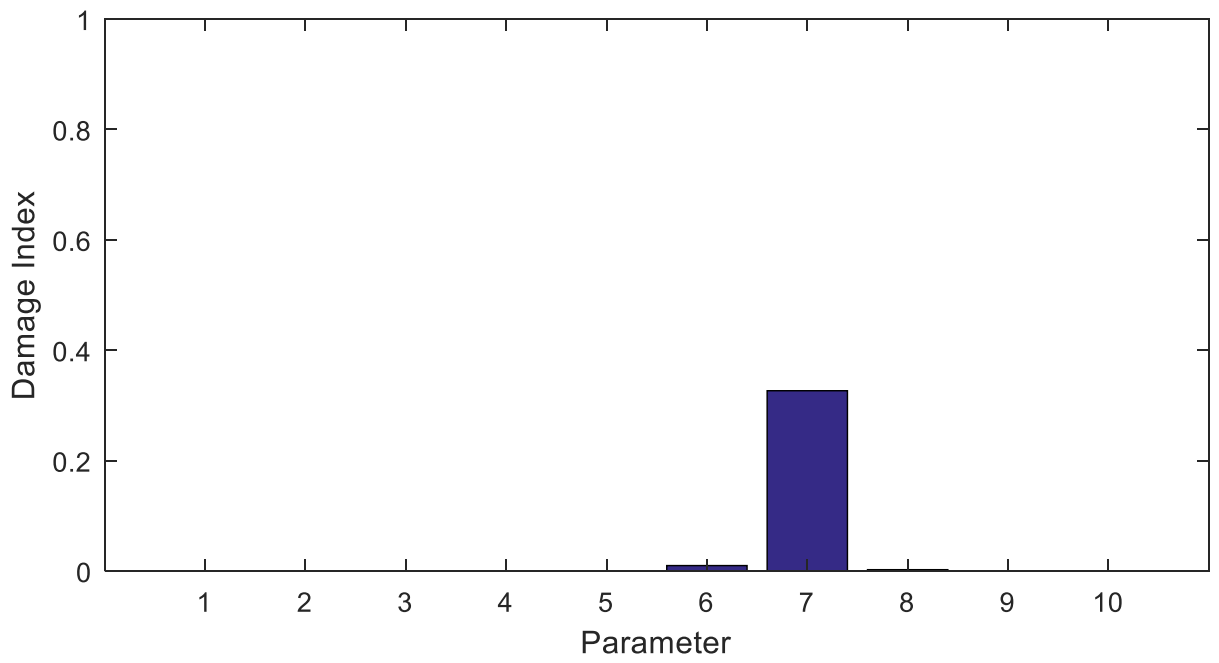

Fig. 12. Damage index for numerical investigation. 
The comparison illustrated in Figure 10 showing the driving point FRFs of the FE model and simulated target data indicates a significant reduction in the discrepancies between the model FRF and the measured FRF after updating. Figure 11 shows that the convergence of the parameters was achieved after 17 iterations. The Young's modulus of each partition remained within $0.4 \%$ of the original value except for P7, which dropped down to $20.1 \mathrm{GPa}$. The damage index calculated using Eq. (2.12) revealed that the region covered by P7 had an overall stiffness reduction of $33.1 \%$ as shown in Figure 12. The results are summarised in Table 1.

Table 1 . Summary of numerical simulated study.

\begin{tabular}{cccccc}
\hline Parameter & $\begin{array}{c}\text { Initial } \\
\text { Parameter } \\
\text { (Mpa) }\end{array}$ & $\begin{array}{c}\text { Updated } \\
\text { Parameter } \\
\text { (Mpa) }\end{array}$ & $\begin{array}{c}\text { Damage } \\
\text { Index }\end{array}$ & $\begin{array}{c}\text { Measured } \\
\text { Parameter } \\
\text { (Mpa) }\end{array}$ & Error \\
\hline P1 & 30,000 & 30,000 & $0.0 \%$ & 30,000 & $0.0 \%$ \\
P2 & 30,000 & 30,000 & $0.0 \%$ & 30,000 & $0.0 \%$ \\
P3 & 30,000 & 30,000 & $0.0 \%$ & 30,000 & $0.0 \%$ \\
P4 & 30,000 & 29,999 & $0.0 \%$ & 30,000 & $0.0 \%$ \\
P5 & 30,000 & 29,999 & $0.0 \%$ & 30,000 & $0.0 \%$ \\
P6 & 30,000 & 29,871 & $0.4 \%$ & 30,000 & $-0.4 \%$ \\
P7 & 30,000 & 20,075 & $33.1 \%$ & 20,000 & $0.4 \%$ \\
P8 & 30,000 & 29,996 & $0.0 \%$ & 30,000 & $0.0 \%$ \\
P9 & 30,000 & 29,999 & $0.0 \%$ & 30,000 & $0.0 \%$ \\
P10 & 30,000 & 29,999 & $0.0 \%$ & 30,000 & $0.0 \%$ \\
BC1 & 50 & 70.08 & - & 70.00 & $0.1 \%$ \\
BC2 & 50 & 39.91 & - & 40.00 & $-0.2 \%$ \\
BC3 & 50 & 8.01 & - & 8.00 & $0.1 \%$ \\
\hline
\end{tabular}

\section{Experimental Validation}

The proposed method was investigated using the experimentally measured data from the jack arch specimen. One healthy case and two damage cases were considered in this experimental validation. The FRF comparisons of these cases are shown in Figure 13. The practical measurements obtained from the dynamic tests had significantly more noise than the numerically simulated measurements. The imperfect boundary conditions were a major source of noise in this investigation. Gaps were observed in the plaster layers used to adhere the specimen to the fixtures. This non-uniformity in the boundary conditions most likely contributed to the splitting of modes identified in the FRF. It has been observed that asymmetry in a structure can lead to peaks splitting in FRFs [24]. This made it difficult to detect all the modes that were identified in the FE model. Figure 13 shows that four modes, including a vertical bending, in-plane bending and two torsion modes could be identified in all three cases. The FRF range used to update the model were selected based on these modes. 


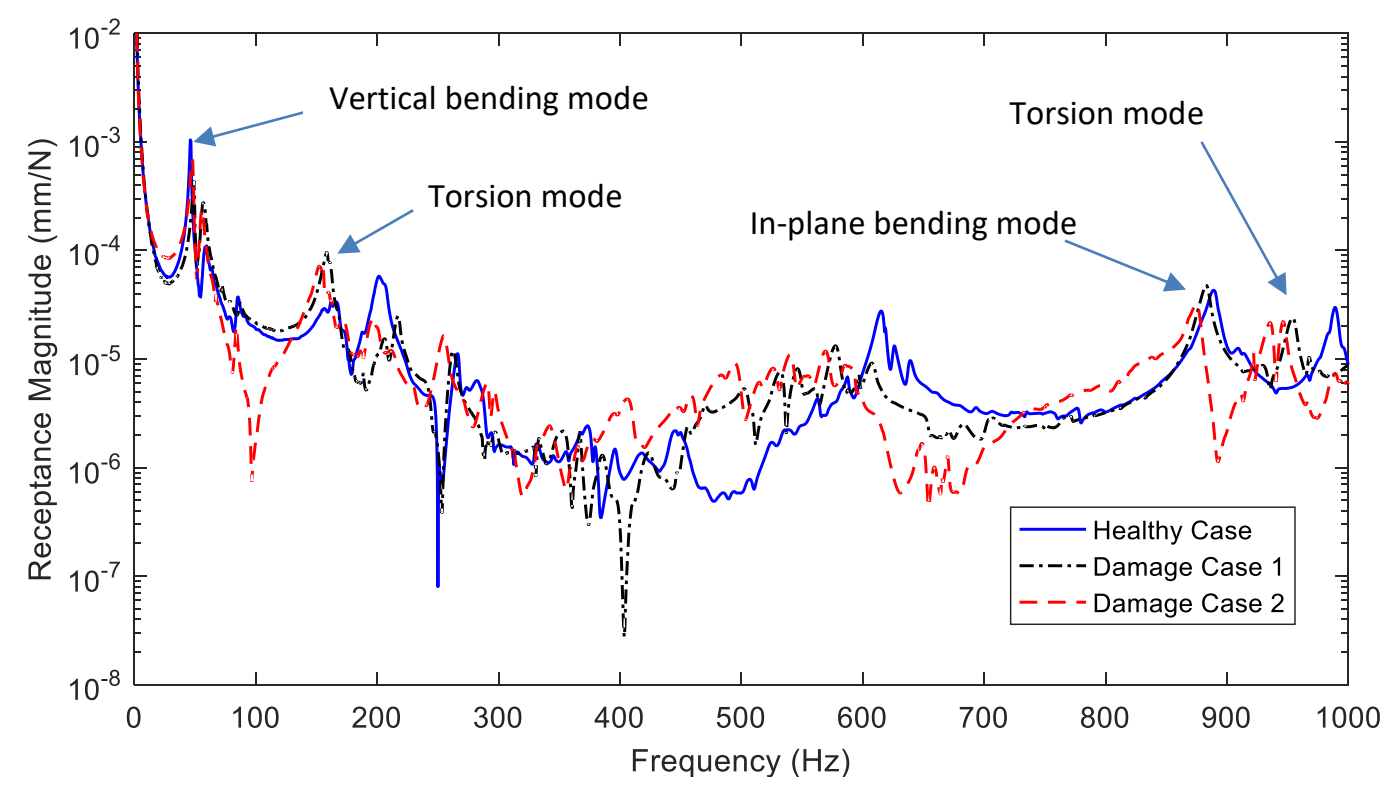

Fig. 13. FRF comparison of healthy and damage cases.

Initial attempts were made to detect damage in the structure using the measurements from the damage cases alone. However, the updated results for P9 and P10 dropped to unrealistically low values. The authors suspect that this is due to the information lost in the FRF used for updating. Multiple peaks were identified in the FRF between $200 \mathrm{~Hz}$ to $800 \mathrm{~Hz}$ in the numerical study, as shown in Figure 10 . However, these peaks could not be identified in Figure 13, with the exception of the mode at $615 \mathrm{~Hz}$ for the healthy case. This mode could not be identified for the damage cases. It is likely that these modes split into multiple modes due to the asymmetry in the cracks. Similarly, the other modes may have not been identified due to the asymmetry in the boundary conditions. To overcome this issue, the number of updating parameters was reduced by dividing the updating procedure into two stages.

The first stage was to update the healthy case of the specimen using four updating parameters including the overall Young's modulus of the specimen and the three boundary condition parameters. The second stage was to update the Young's modulus of the 10 partitions of the jack arch specimen only i.e., P1 to P10. The Young's modulus of the specimen and boundary conditions identified in the first stage were, respectively, used as the healthy Young's modulus and fixed boundary condition parameters in the second stage. It was assumed that the boundary conditions would not change between different cases. The FRFs of the healthy case were cleaned by synthesising the FRFs using the modal data obtained experimentally and applying them to Eq. (2.6). The Rayleigh damping parameters of $\alpha=5.95$ and $\beta=1.1 \times 10^{-6}$ were assumed based on the damping ratios identified from the first and last mode of interest. A comparison of the synthesised and measured driving point FRF is shown in Figure 14. The first vertical bending and in-plane bending mode, and the second torsion mode were used to update the model in its healthy state. The first torsion mode identified at $167 \mathrm{~Hz}$ was excluded from the updating procedure, as the damping properties of this mode could not be represented by the Rayleigh damping parameters used in the FE model. The peak identified at $615 \mathrm{~Hz}$ was also a torsion mode that was removed from the model updating procedure, as it had a significantly smaller peak for the measured FRF than the one identified from the FE model. Further, this mode could not be clearly identified for the damage cases. Hence it was removed from the model updating process. For the healthy case, the frequencies considered for updating the model included 0 $\mathrm{Hz}$ to $100 \mathrm{~Hz}$ and $800 \mathrm{~Hz}$ to $1,000 \mathrm{~Hz}$. The frequency resolution was $2 \mathrm{~Hz}$ for the considered range. 


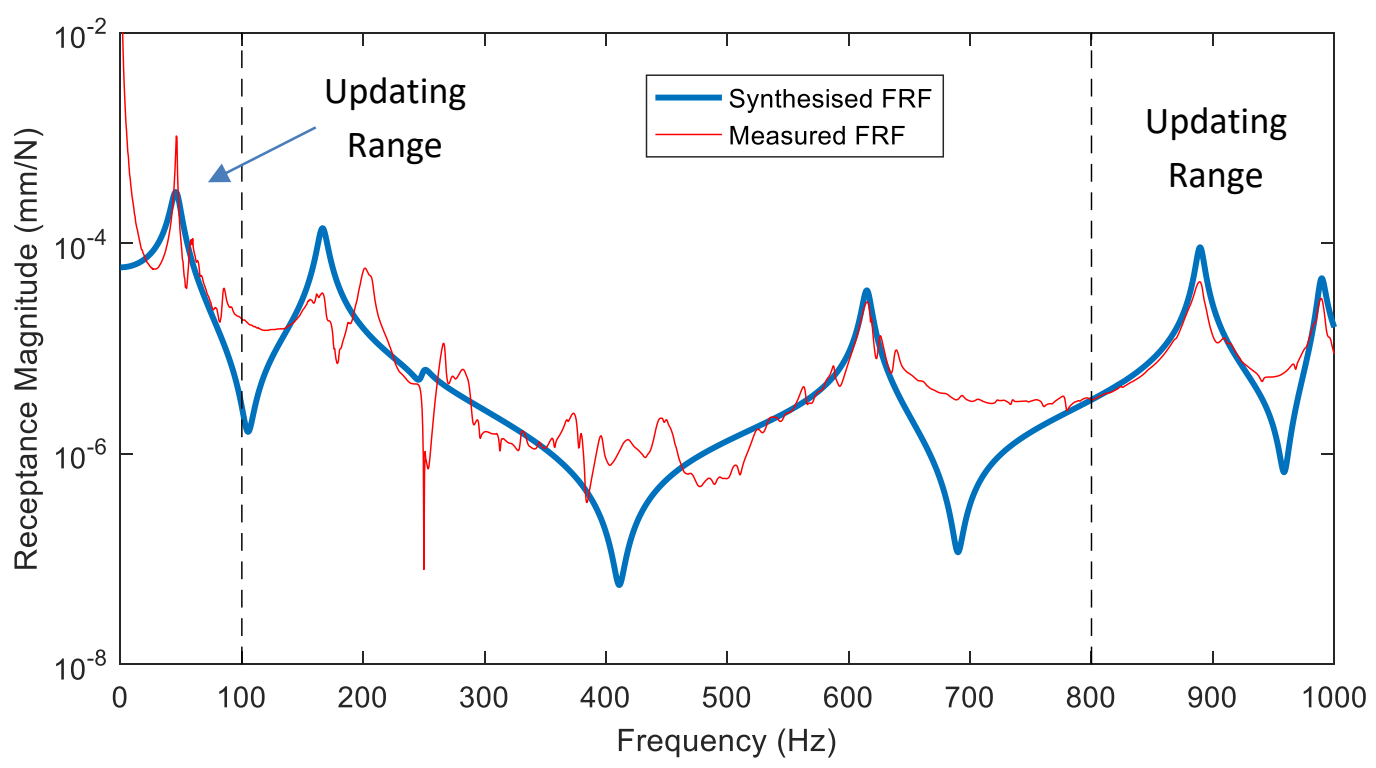

Fig. 14. Driving point synthesised FRF versus driving point measured FRF for the healthy case.

Similarly, to the numerical case, the FE model was reduced to the 60 DOF linkage model shown in Figure 8, which was then used to expand the mode shapes of the 11 measured DOFs as shown in Figure 15. The interpolated FRFs were synthesised using the expanded modal data and used to form the FRF sensitivity matrix, which contained of 4 columns corresponding to the updating parameters and 18,240 rows to accommodate the 152 measured frequencies, 60 DOFs and the splitting of the real and imaginary components of the FRF. A lower limit of $15 \mathrm{MPa}$ was placed for the boundary condition parameters to stop them from falling to unrealistic values. The linear least-squares problem expressed in Eq. (2.8) was solved and this procedure was repeated until 20 iterations were reached. The results in Figure 16 show that the updated FRF is a closer representation of the measured FRF than the initial FRF. The iterative change of parameters is shown in Figure 17 and the results of updating the healthy specimen is summarised in Table 2 . The next step of this investigation was to update the partitions of the FE model using the FRFs measured from the damaged specimen.

Table 2. Summary of updating of healthy specimen.

\begin{tabular}{ccc}
\hline Parameter & Initial Parameter $(\mathrm{MPa})$ & Updated Parameter $(\mathrm{MPa})$ \\
\hline Concrete Young's Modulus & 30,000 & 32,721 \\
Boundary Condition 1 & 50 & 39.50 \\
Boundary Condition 2 & 50 & 35.85 \\
Boundary Condition 3 & 50 & 17.49 \\
\hline
\end{tabular}



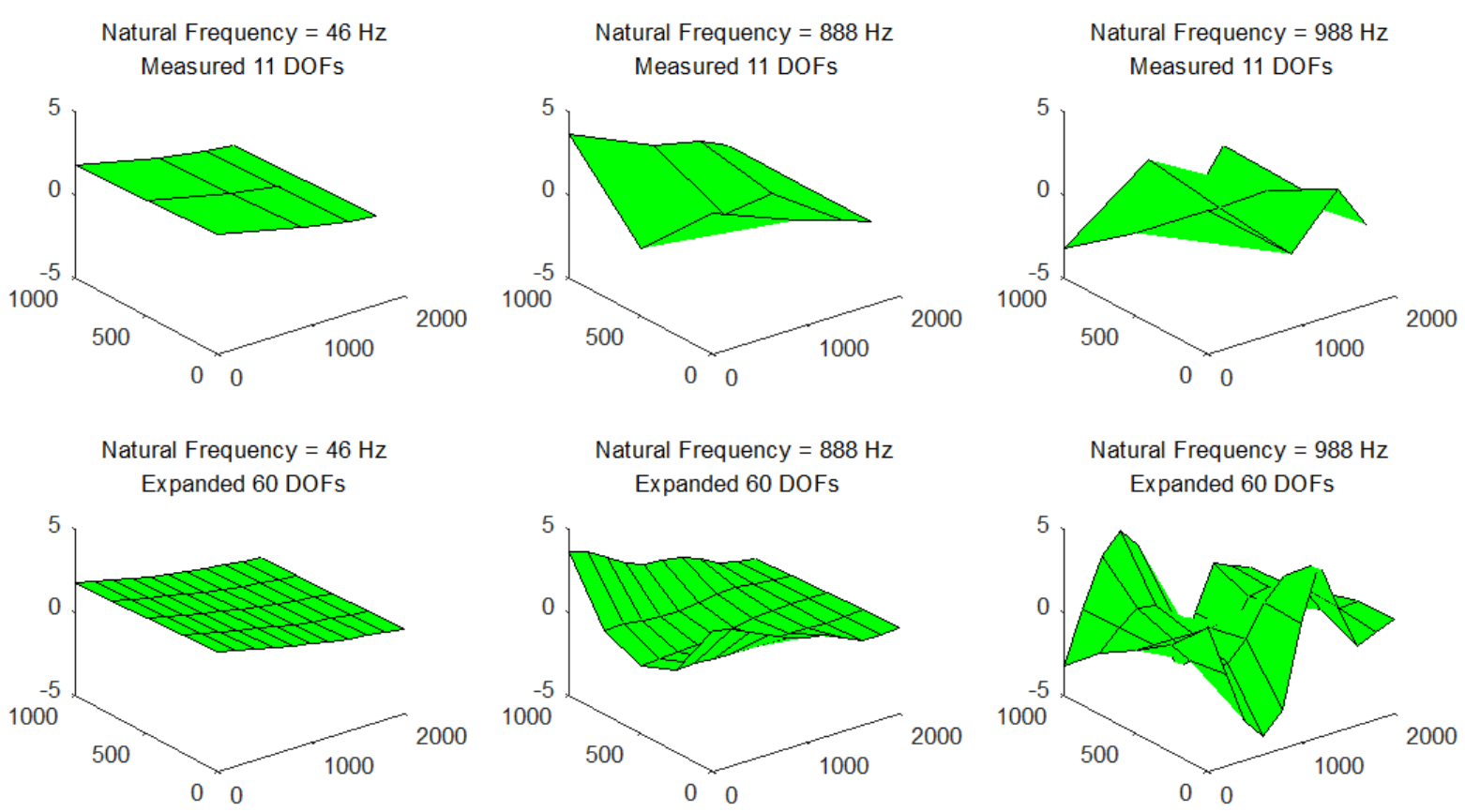

Fig. 15. Examples of mode shapes expanded from 11 DOF to 60 DOF for the experimental healthy case.
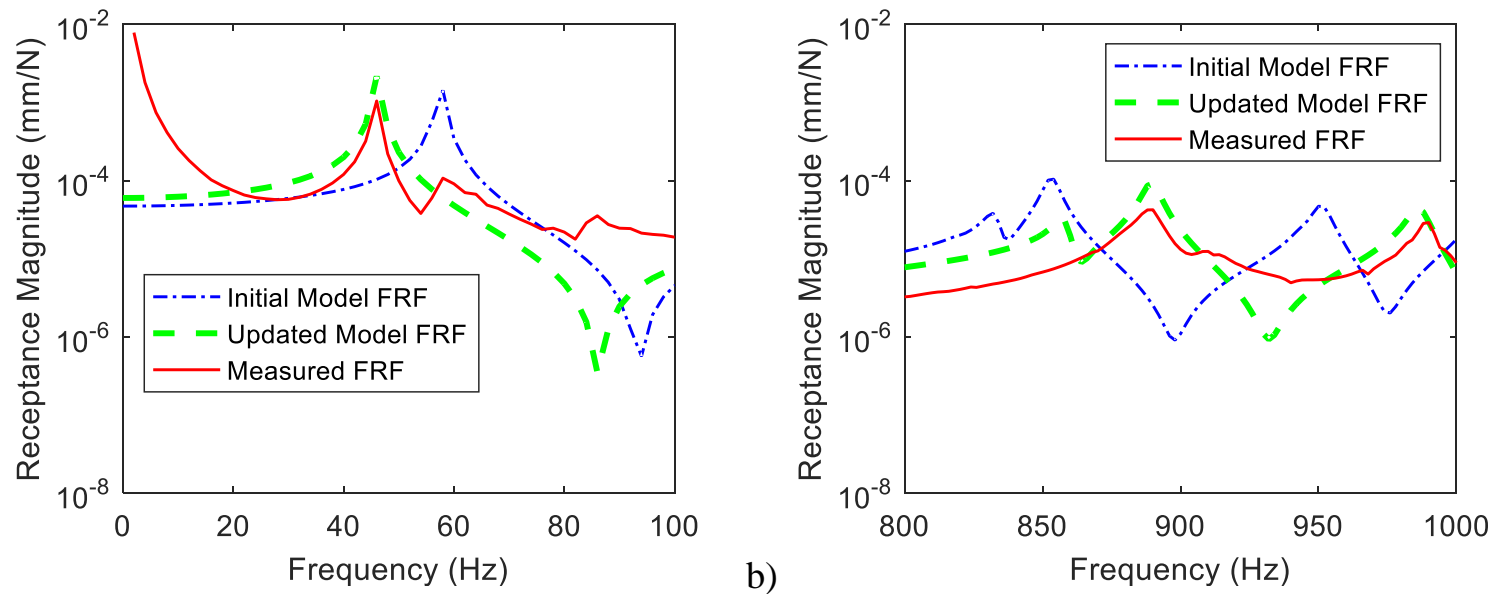

Fig. 16. Driving point FRF before and after updating for the healthy case at frequencies (a) $0 \mathrm{~Hz}$ to $100 \mathrm{~Hz}$ (b) $800 \mathrm{~Hz}$ to $1000 \mathrm{~Hz}$. 


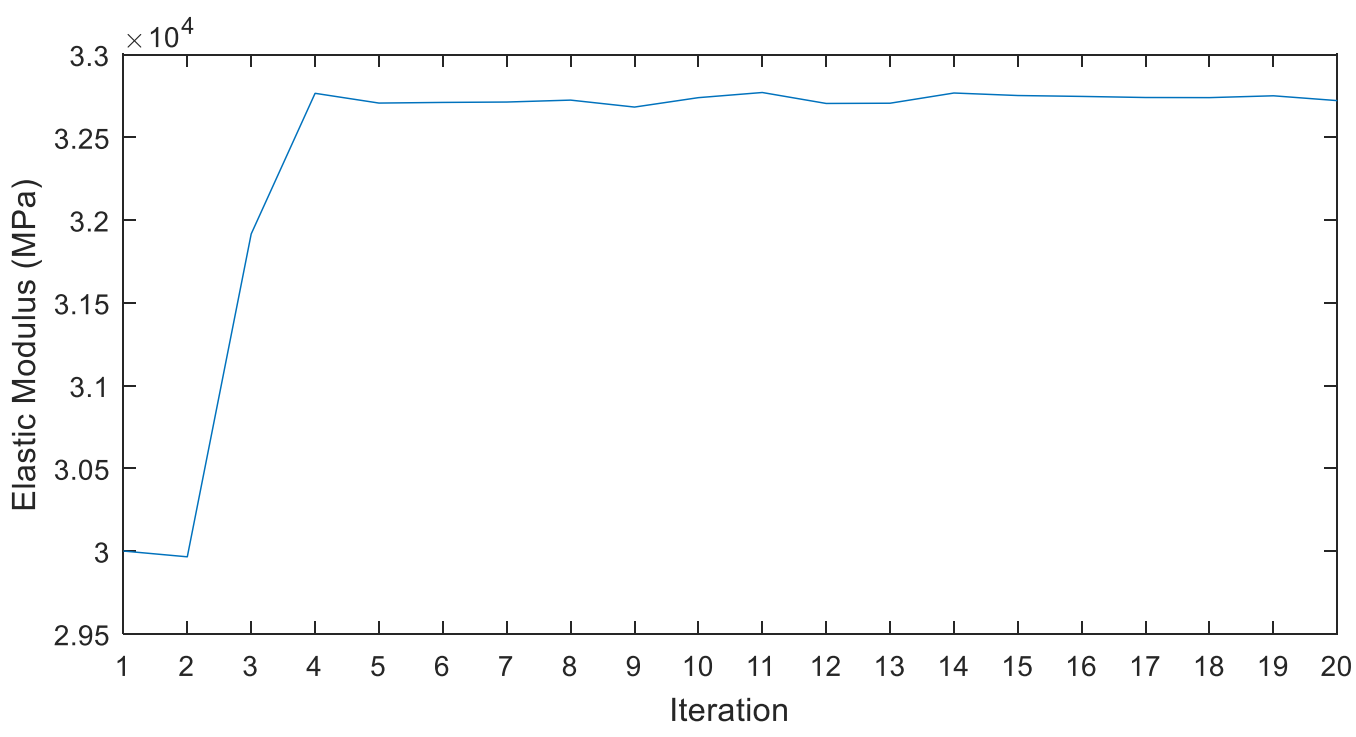

a)

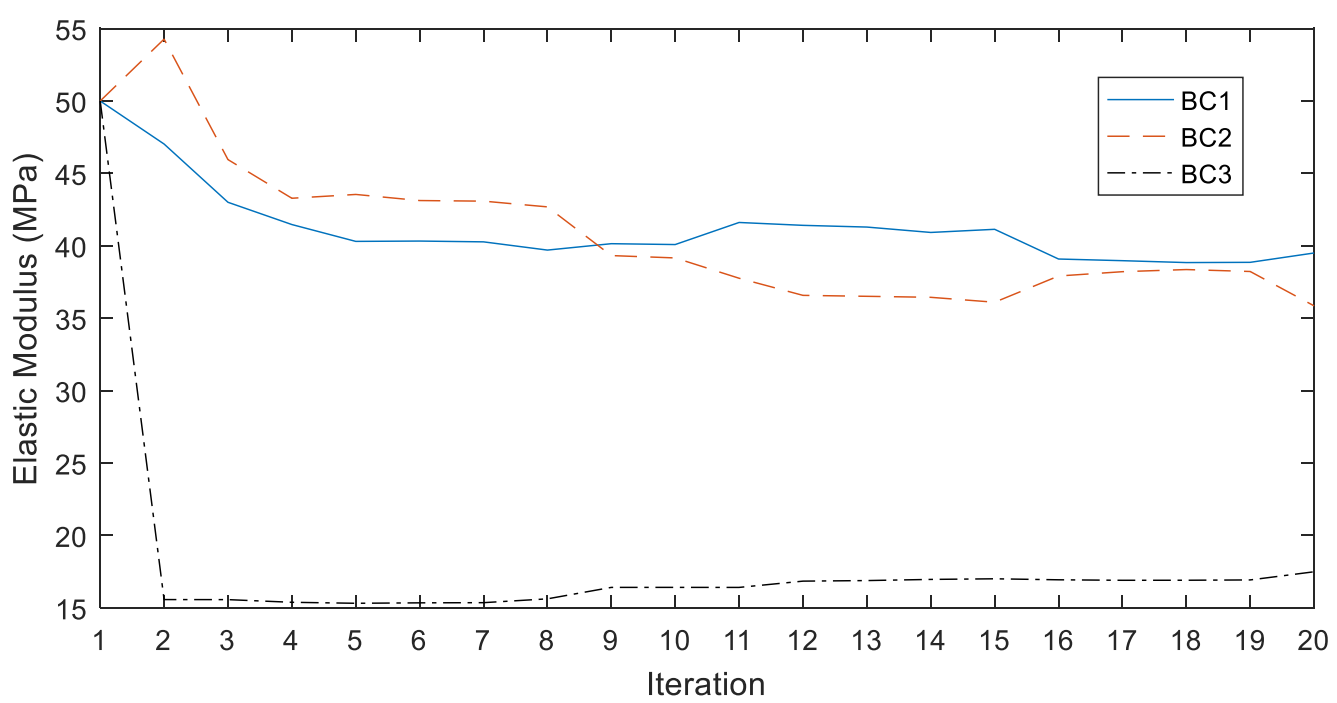

b)

Fig. 17. Iterative changes of parameters of the healthy case for the Young's modulus of the (a) concrete specimen and (b) boundary conditions adhesives.

The next step of this procedure was to identify the damage location and severity using the data measured in the first damage case. As with the healthy case, the FRFs were cleaned by synthesising the FRFs using modal data obtained experimentally. Rayleigh damping was assumed based on the damping ratios of the first and last modes considered in this investigation leading to the parameters $\alpha=48.6$ and $\beta=-2.5 \times 10^{-7}$. A comparison between the synthesised FRF and the measured FRF is shown in Figure 18. The frequency range chosen for updating were based on the four modes identified in Figure 13. In this case, the frequency range chosen was $0 \mathrm{~Hz}$ to $200 \mathrm{~Hz}$ and $800 \mathrm{~Hz}$ to $1,000 \mathrm{~Hz}$ with a resolution of $2 \mathrm{~Hz}$. In each iteration of the model updating procedure, the model was reduced to the linkage model, which was used to expand the measured mode shapes. Examples of expanded mode shapes of the first damaged case are illustrated in Figure 19. The expanded mode shapes were used to synthesise the interpolated FRFs, which was used, in combination with the linkage model, to form the sensitivity matrix to update the parameters of the FE model. The sensitivity matrix contained of ten columns, corresponding to the Young's modulus of the ten sections 
of the jack arch specimen and contained 24,240 rows to accommodate the 202 measured frequencies, 60 DOFs and splitting the real and imaginary components of the FRF.

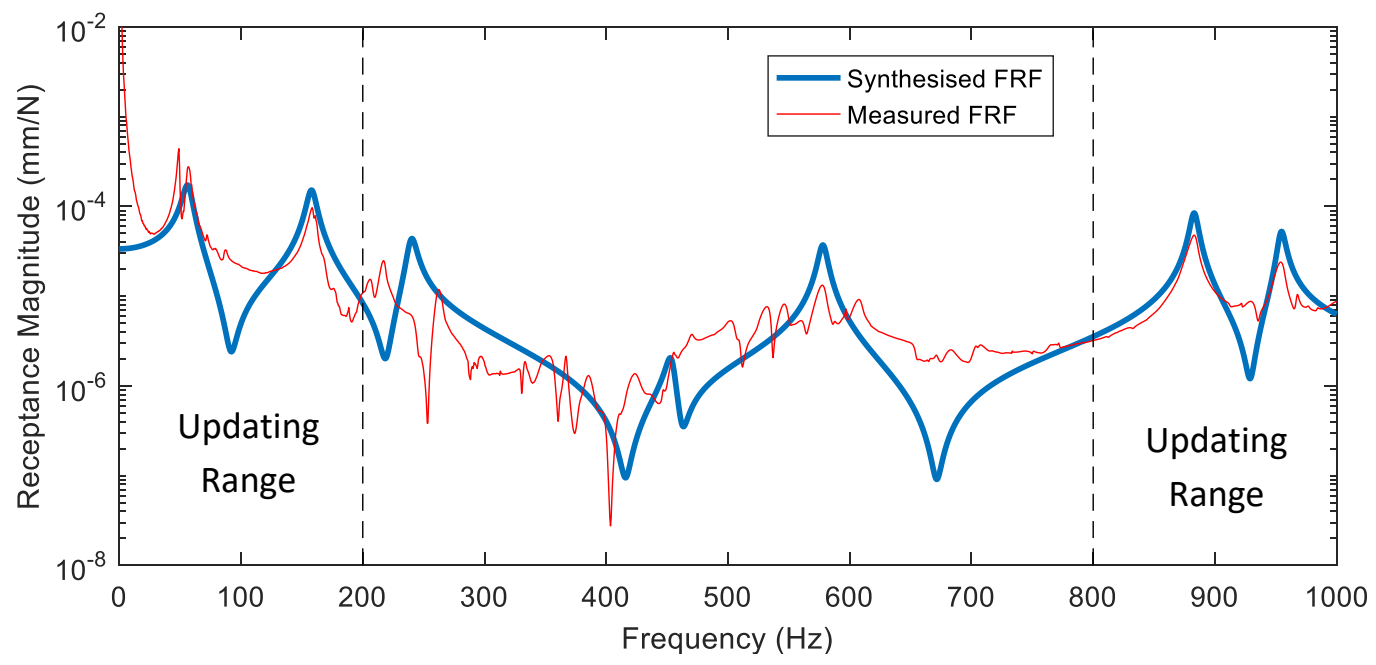

Fig. 18. Driving point synthesised FRF vs driving point measured FRF for Damage Case 1.
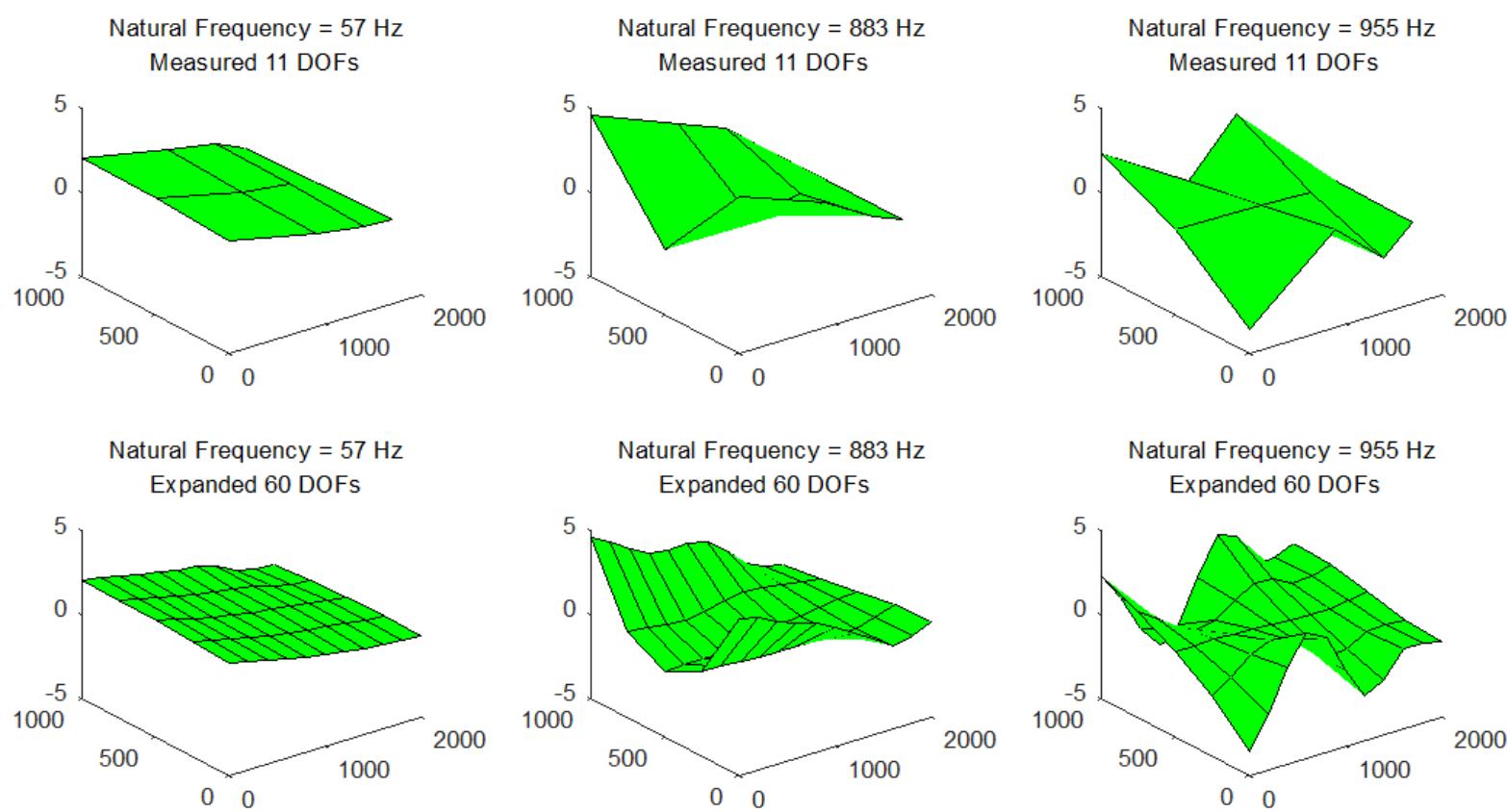

Fig. 19. Examples of mode shapes expanded from 11 DOF to 60 DOF for Damage Case 1. 

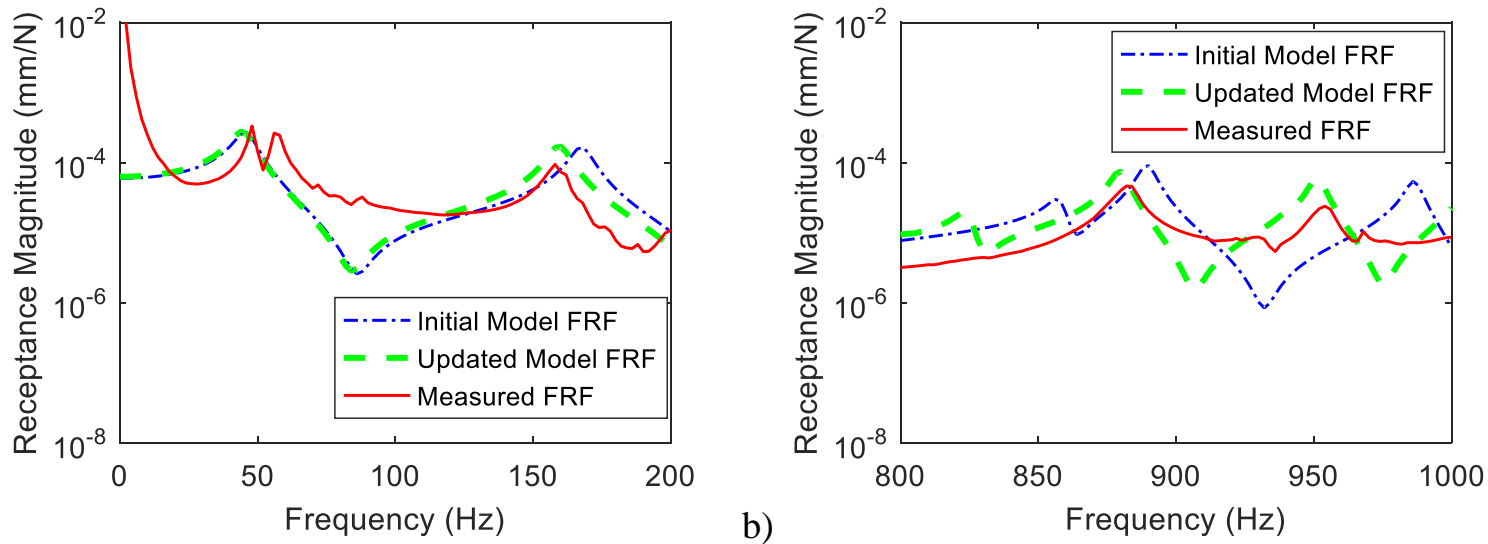

Fig. 20. Driving point FRF before and after updating for Damage Case 1 at frequencies (a) $0 \mathrm{~Hz}$ to $200 \mathrm{~Hz}$ (b) $800 \mathrm{~Hz}$ to $1,000 \mathrm{~Hz}$

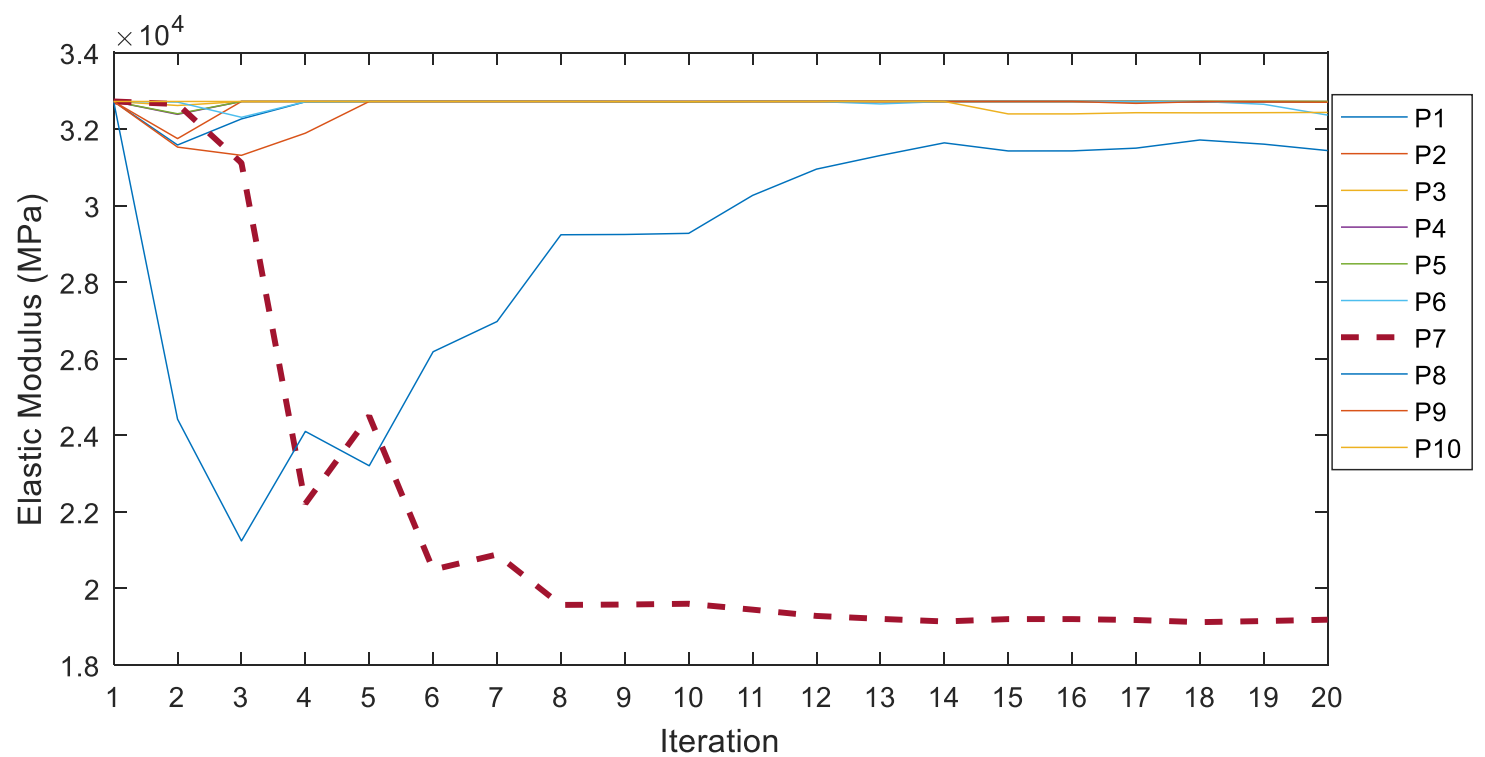

Fig. 21. Convergence of updating parameters for Damage Case 1.

Following the model updating procedure, there was a closer agreement between the model FRF and the measured FRF as shown in Figure 20. The model updating procedure was stopped once the maximum of 20 iterations were reached. The updated parameter P7 showed a clear decrease in its Young's modulus value in comparison to the other partitions of the specimen. This can be seen in Figure 21. Based on the updated information, the damage index was calculated. The results shown in Figure 22 and Table 3 indicate that $\mathrm{P} 7$ had a stiffness reduction of $41.4 \%$, which was significantly larger than the other partitions of the specimen. These results agree with the crack visually identified in Figure 6, which was located between $1,300 \mathrm{~mm}$ and 1,400 $\mathrm{mm}$ from the front of the specimen and lies within the region covered by $\mathrm{P} 7$. 


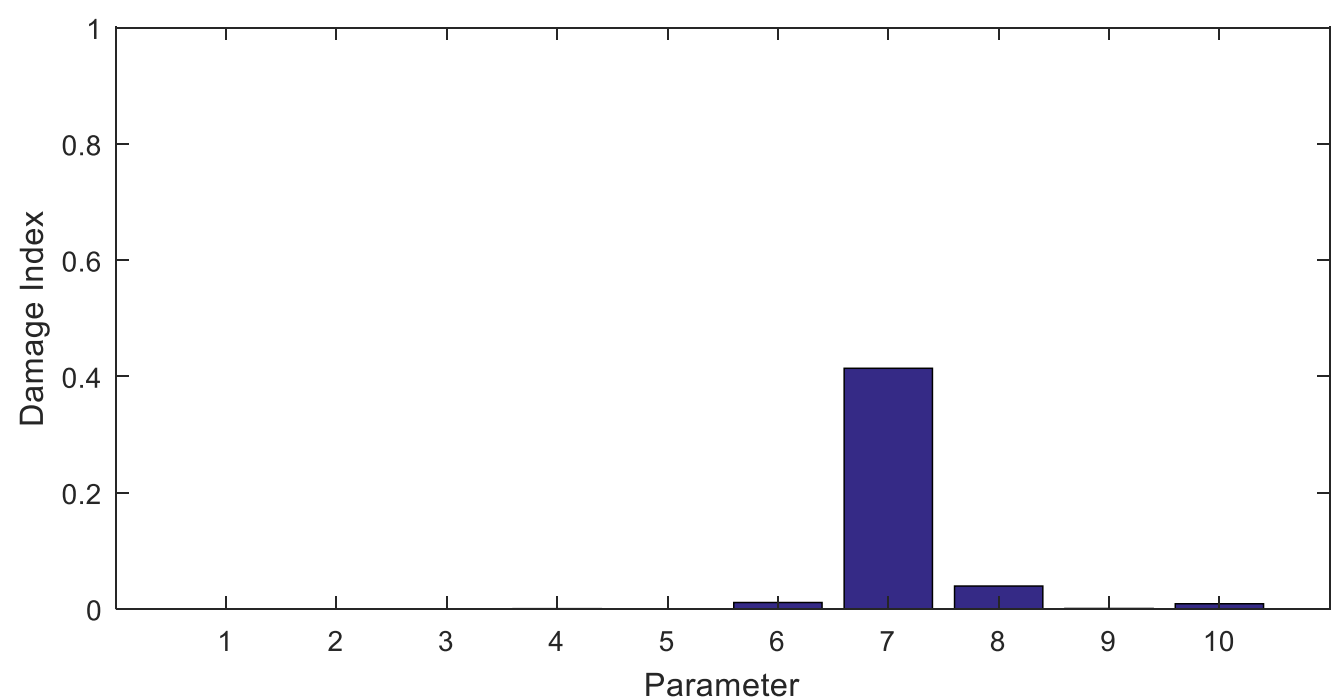

Fig. 22. Damage index for Damage Case 1 in the experimental investigation.

Table 3. Summary of updating for Damage Case 1.

\begin{tabular}{cccc}
\hline Parameter & Initial Parameter (Mpa) & Updated Parameter (Mpa) & Damage Index \\
\hline P1 & 32,721 & 32,721 & $0.0 \%$ \\
P2 & 32,721 & 32,717 & $0.0 \%$ \\
P3 & 32,721 & 32,721 & $0.0 \%$ \\
P4 & 32,721 & 32,710 & $0.0 \%$ \\
P5 & 32,721 & 32,719 & $0.0 \%$ \\
P6 & 32,721 & 32,365 & $1.1 \%$ \\
P7 & 32,721 & 19,181 & $41.4 \%$ \\
P8 & 32,721 & 31,438 & $3.9 \%$ \\
P9 & 32,721 & 32,700 & $0.1 \%$ \\
P10 & 32,721 & 32,435 & $0.9 \%$ \\
\hline
\end{tabular}

The second damage case was the next case to be tested in the model updating procedure. As with the previous two experimental cases, cleaned FRFs were synthesised using the modal data obtained experimentally. The Rayleigh damping values of $\alpha=82.8$ and $\beta=1.0 \times 10^{-6}$ were used based on the first and last mode considered for the second damaged case. Figure 23 illustrates the comparison between the synthesised and measured driving point FRFs. A frequency range of $0 \mathrm{~Hz}$ to $200 \mathrm{~Hz}$ and $800 \mathrm{~Hz}$ to $1,000 \mathrm{~Hz}$ with a resolution of $2 \mathrm{~Hz}$ was chosen to include the modes identified in Figure 13. The model updating procedure described for the previous experimental cases was also used for this case. Figure 24 shows that the model FRF has become a closer representation to the measured FRF after updating. Parameter convergence is shown in Figure 25 and was stopped after 20 iterations were reached. The Young's modulus of P7 dropped to a value of $14.9 \mathrm{GPa}$ during this model updating procedure. Based on these results, the damage index was calculated and can be seen in Figure 26 and Table 4. A stiffness reduction of $54.4 \%$ could be observed in P7 as a result of the crack after the second load. 


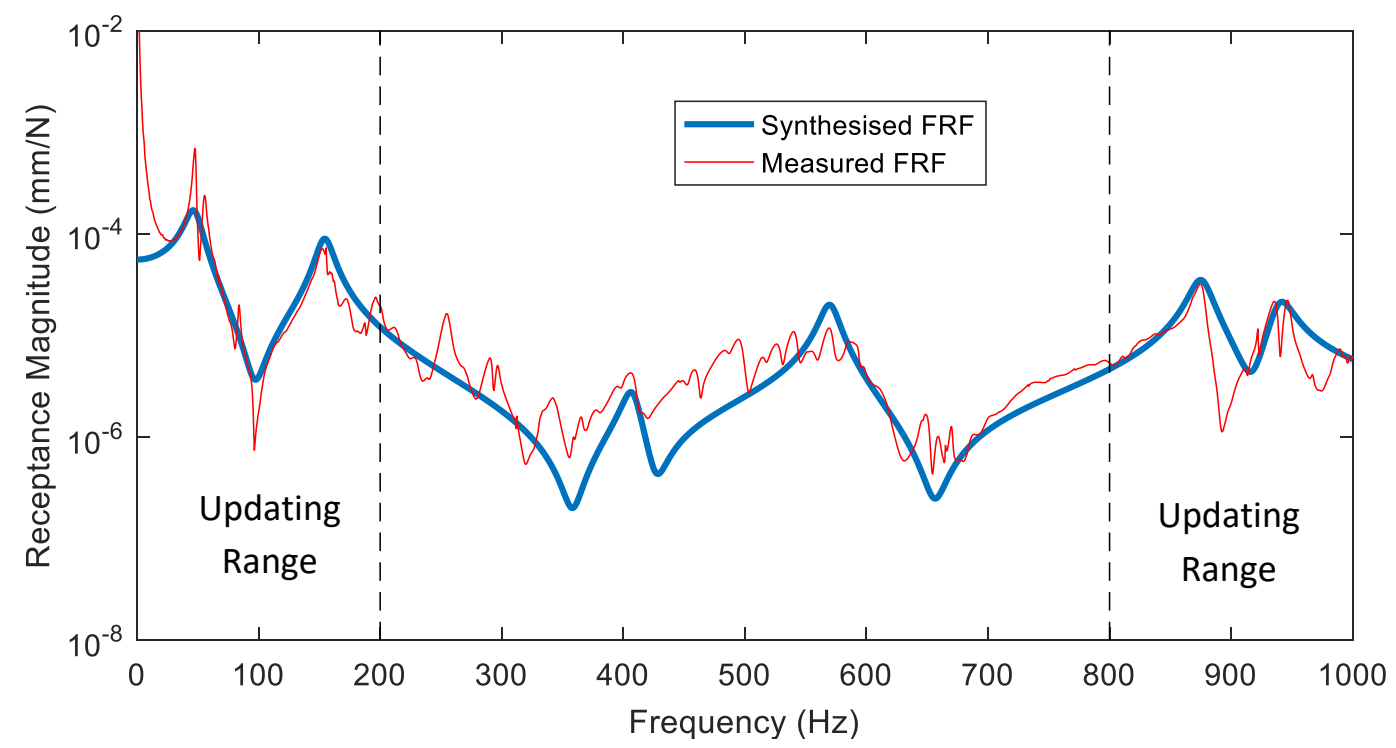

Fig. 23. Driving point synthesised FRF vs driving point measured FRF for Damage Case 2.
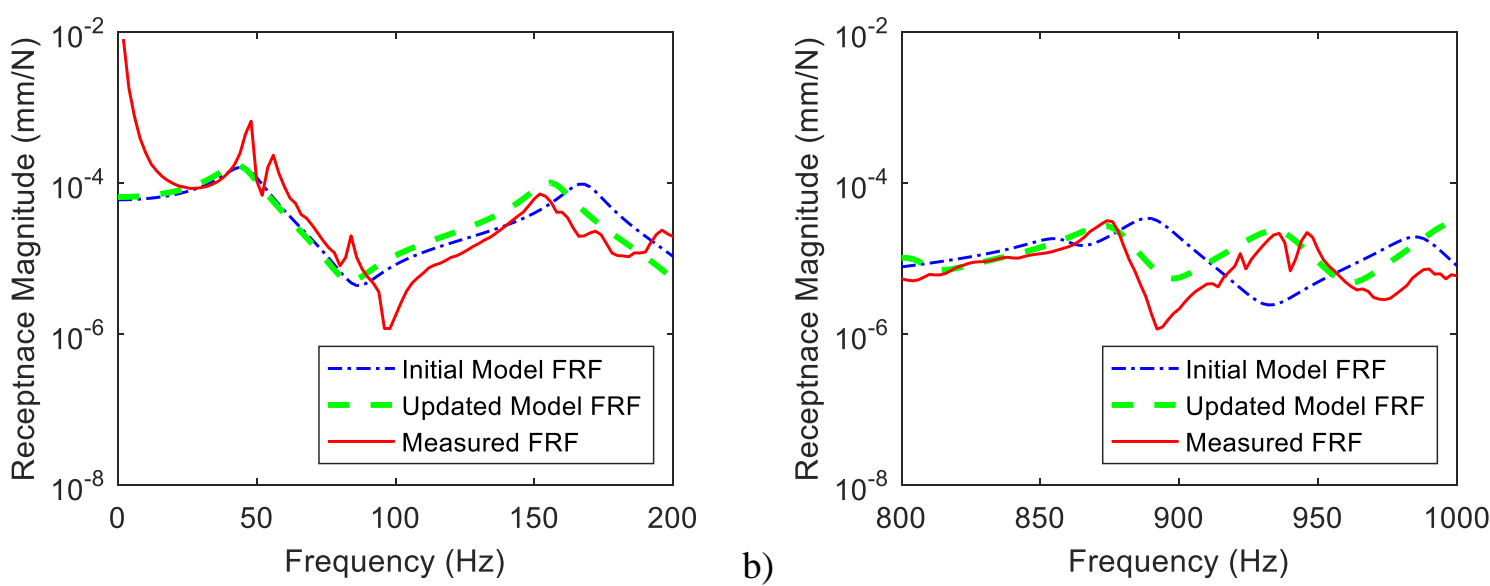

Fig. 24. Driving point FRF before and after updating for Damage Case 2 at frequencies (a) $0 \mathrm{~Hz}$ to $200 \mathrm{~Hz}$ (b) $800 \mathrm{~Hz}$ to $1,000 \mathrm{~Hz}$

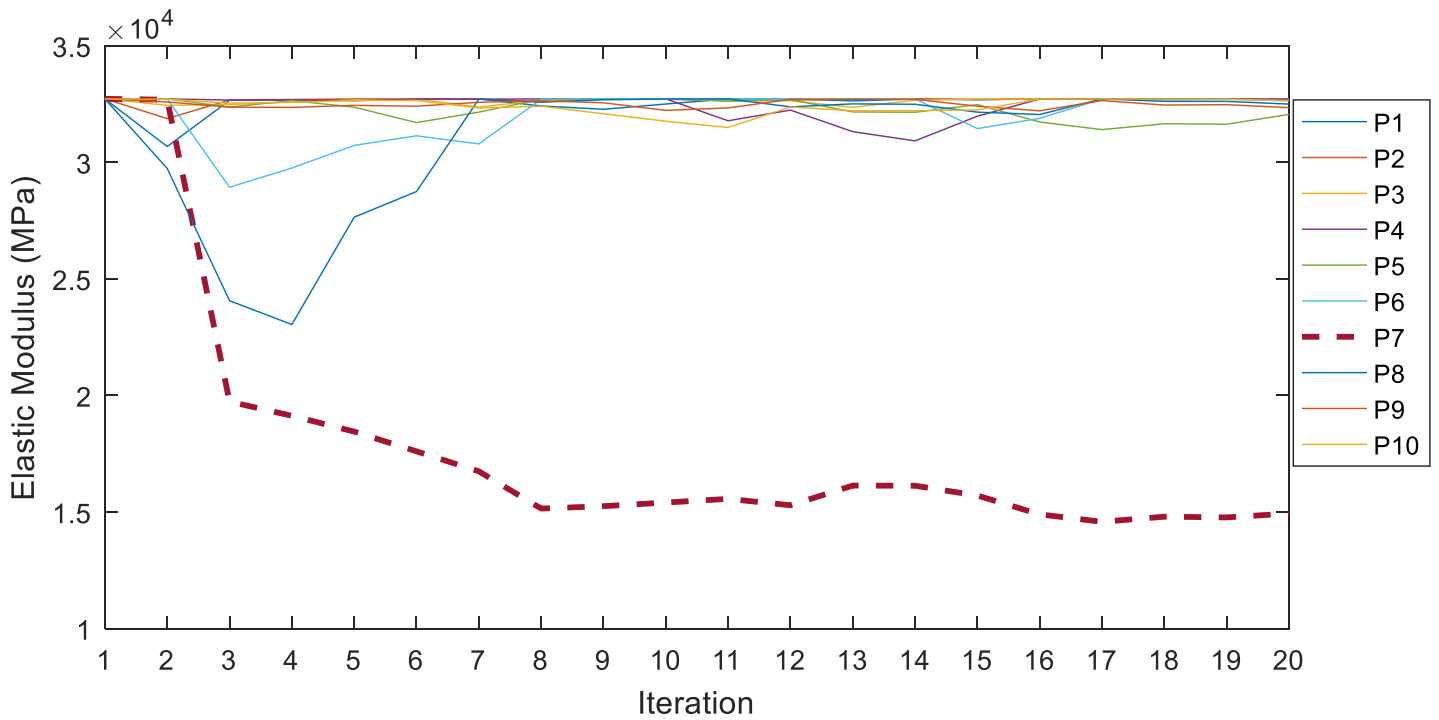

Fig. 25. Convergence of updating parameters for Damage Case 2. 


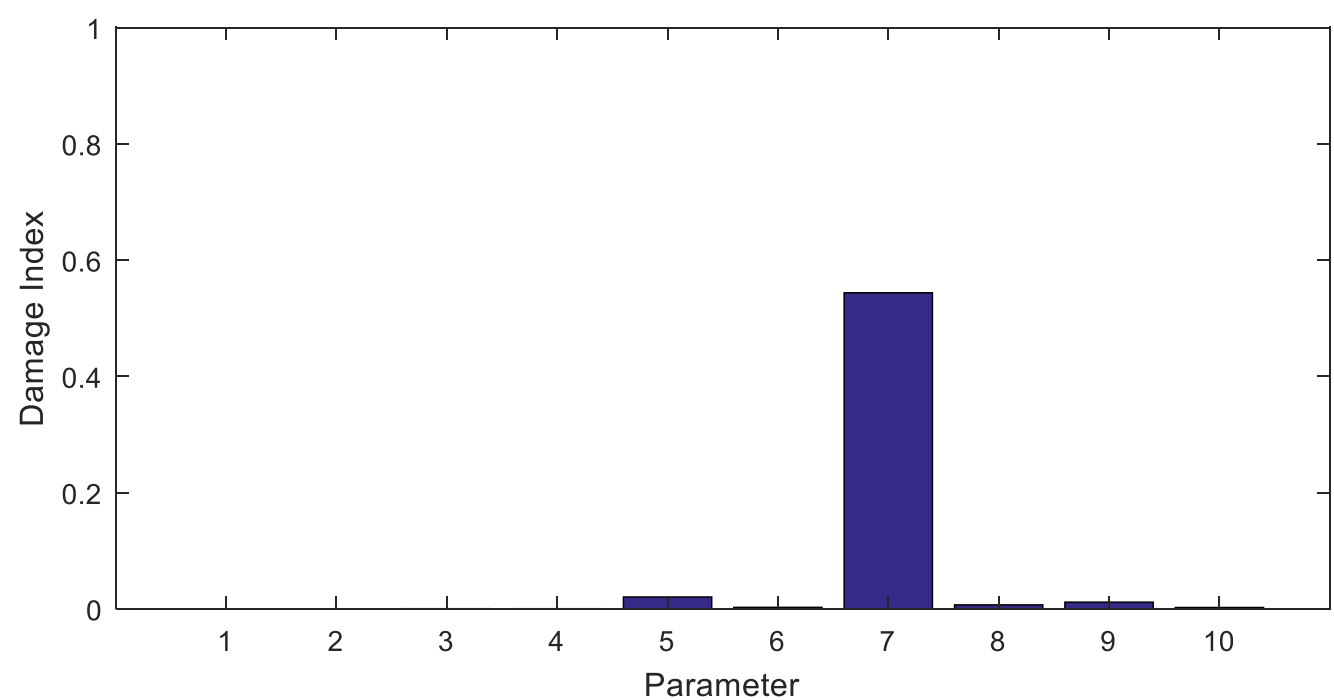

Fig. 26. Damage index for Damage Case 2 in the experimental investigation.

Table 4. Summary of updating of Damage Case 2.

\begin{tabular}{cccc}
\hline Parameter & Initial Parameter (Mpa) & Updated Parameter (Mpa) & Damage Index \\
\hline P1 & 32,721 & 32,717 & $0.0 \%$ \\
P2 & 32,721 & 32,717 & $0.0 \%$ \\
P3 & 32,721 & 32,713 & $0.0 \%$ \\
P4 & 32,721 & 32,713 & $0.0 \%$ \\
P5 & 32,721 & 32,055 & $2.0 \%$ \\
P6 & 32,721 & 32,638 & $0.3 \%$ \\
P7 & 32,721 & 14,934 & $54.4 \%$ \\
P8 & 32,721 & 32,498 & $0.7 \%$ \\
P9 & 32,721 & 32,345 & $1.1 \%$ \\
P10 & 32,721 & 32,646 & $0.2 \%$ \\
\hline
\end{tabular}

Overall, the proposed method could detect the stiffness reduction in partition P7 due to the presence of the crack located within that region using a limited sensor array. Two damage cases were tested with P7 being found to have a stiffness reduction of $41.4 \%$ and $54.4 \%$ for Damage Case 1 and Damage Case 2, respectively. This is consistent with the observations of the crack identified on the specimen approximately $1,350 \mathrm{~mm}$ from its front. After the first static load, the crack was recorded to have a depth of $275 \mathrm{~mm}$. The specimen was loaded again and the crack length increased to $300 \mathrm{~mm}$. The investigation into the correlation between the crack depth and stiffness reduction was outside the scope of this paper. However, future research will involve the investigation into the correlation between the stiffness reduction of a partition and crack properties in a similar manner to the research conducted by Sinha et al. [7]. The authors of that study made small modifications to the local flexibility in Euler-Bernoulli beam elements to estimate crack locations and sizes.

In SHM, it is impossible to apply a sensor on every point corresponding to the DOFs in the FE model. The linkage model serves as an intermediary to link the FE model to the spatially limited measured data. This technique can be used in real world applications where the spatial limitation of sensors is an issue. 


\section{Conclusions}

The presented study investigated a method to detect the location and severity of damage in a jack arch specimen by updating a linkage FE model using the FRF sensitivity method. In the developed method, the specimen was first divided into partitions, then the Young's modulus of each partition was updated to identify the stiffness reduction in each section. The numerical validation showed that the method could identify the location and severity of damage within 17 iterations. However, the experimental investigation proved to be more challenging. In general, the modes between $200 \mathrm{~Hz}$ and $800 \mathrm{~Hz}$ could not be clearly identified. A mode at $615 \mathrm{~Hz}$ was identified from the healthy case. However, the mode appeared to have been split into multiple peaks for the damage cases, potentially due to the asymmetry in the crack. Thus it was necessary to reduce the number of updating parameters by diving the procedure into two stages. For the healthy case of the experimental investigation, four parameters including the overall Young's modulus of the specimen, and three parameters based on the boundary conditions of the specimen were updated. The updated overall Young's modulus of the specimen was treated as the healthy Young's modulus and the updated boundary condition parameters were fixed for the damaged case. Then, the Young's moduli of the 10 partitions of the specimen were used as the updating parameters in the model updating procedure for the damaged cases. The results correctly located the damage in partition P7 with a stiffness reduction value of $41.4 \%$ after the first load. For the second damage case, it identified an increased stiffness reduction value of $54.4 \%$ at P7. The method is a practical approach to interpolating data to identify the location and severity of damage for real SHM systems that contain a limited numbers of measurement sensors.

\section{Acknowledgements}

This research is supported by an Australian Government Research Training Program Scholarship. The authors also wish to thank the Road and Maritime Services (RMS) in New South Wales, Australia and NICTA for provision of the support and testing facilities for this research work. NICTA is funded by the Australian Government through the Department of Communications and the Australian Research Council through the ICT Centre of Excellence Program. CSIRO's Digital Productivity business unit and NICTA have joined forces to create digital powerhouse Data61.

\section{References}

1. Doebling, Scott W., Charles R. Farrar, and Michael B. Prime. "A summary review of vibration-based damage identification methods." Shock and vibration digest 30.2 (1998): 91-105.

2. Sohn, Hoon, et al. "A review of structural health monitoring literature: 1996-2001." Los Alamos National Laboratory (2003).

3. Kang, Fei, Jun-Jie Li, and Qing Xu. "Damage detection based on improved particle swarm optimization using vibration data." Applied Soft Computing 12.8 (2012): 2329-2335.

4. Hao, Hong, and Yong Xia. "Vibration-based damage detection of structures by genetic algorithm." Journal of computing in civil engineering 16.3 (2002): 222-229.

5. Perera, Ricardo, and Ronald Torres. "Structural damage detection via modal data with genetic algorithms." Journal of Structural Engineering 132.9 (2006): 1491-1501.

6. Mottershead, John E., Michael Link, and Michael I. Friswell. "The sensitivity method in finite element model updating: a tutorial." Mechanical systems and signal processing 25.7 (2011): 2275-2296.

7. Sinha, Jyoti K., M. I. Friswell, and S. Edwards. "Simplified models for the location of cracks in beam structures using measured vibration data." Journal of Sound and vibration 251.1 (2002): 13-38. 
8. Shi, Z.Y., S. S. Law, and L.M. Zhang. "Structural damage detection from modal strain energy change." Journal of engineering mechanics 126.12 (2000): 1216-1223.

9. Mousavi, Mohsen, and Amir H. Gandomi. "A hybrid damage detection method using dynamicreduction transformation matrix and modal force error." Engineering Structures 111 (2016): 425-434.

10. Hansen, J. B., et al. "A new scenario-based approach to damage detection using operational modal parameter estimates." Mechanical Systems and Signal Processing 94 (2017): 359-373.

11. Sun, Hao, and Oral Büyüköztürk. "Probabilistic updating of building models using incomplete modal data." Mechanical Systems and Signal Processing 75 (2016): 27-40.

12. Weng, Shun, et al. "Dynamic condensation approach to the calculation of eigensensitivity." Computers \& Structures 132 (2014): 55-64.

13. Au, F. T. K., et al. "Structural damage detection based on a micro-genetic algorithm using incomplete and noisy modal test data." Journal of Sound and Vibration 259.5 (2003): 1081-1094.

14. Entezami, Alireza, Hashem Shariatmadar, and Hassan Sarmadi. "Structural damage detection by a new iterative regularization method and an improved sensitivity function." Journal of Sound and Vibration 399 (2017): 285-307.

15. O'Callahan, John, Peter Avitabile, and Robert Riemer. "System equivalent reduction expansion process (SEREP)." Proceedings of the 7th international modal analysis conference. Vol. 1. Schnectady, NY: Union College, 1989.

16. Alamdari, Mehrisadat Makki, et al. "Nonlinear joint model updating in assembled structures." Journal of Engineering Mechanics 140.7 (2013): 04014042.

17. Dos Santos, JV Araujo, et al. "Structural damage identification in laminated structures using FRF data." Composite Structures 67.2 (2005): 239-249.

18. Mustapha, S., et al. "Pattern recognition based on time series analysis using vibration data for structural health monitoring in civil structures." Electronic Journal of Structural Engineering (2015).

19. Nguyen, V. V., et al. "Damage identification of a concrete arch beam based on frequency response functions and artificial neural networks." Electron J Struct Eng 14.1 (2015): 75-84.

20. Friswell, Michael, and John E. Mottershead. Finite element model updating in structural dynamics. Vol. 38. Springer Science \& Business Media, 2013.

21. Maia, N. M. M., and J. M. M. Silva. Theoretical and Experimental Modal Analysis. Hertfordshire: Research Studies Press Ltd., 1998. Print.

22. Lin, R. M., and D. J. Ewins. "Analytical model improvement using frequency response functions." Mechanical Systems and Signal Processing 8.4 (1994): 437-458.

23. Imregun, M., W. J. Visser, and D. J. Ewins. "Finite element model updating using frequency response function data: I. Theory and initial investigation." Mechanical Systems and Signal Processing 9.2 (1995): 187-202.

24. Davini, C., A. Morassi, and N. Rovere. "Modal analysis of notched bars: tests and comments on the sensitivity of an identification technique." Journal of Sound and Vibration 179.3 (1995): 513-527. 